\title{
Lateral-Directional Parameter Estimation on the X-48B Aircraft Using an Abstracted, Multi-Objective Effector Model
}

\author{
Nalin A. Ratnayake*, Erin R. Waggoner ${ }^{\dagger}$, and Brian R. Taylor ${ }^{\ddagger}$ \\ NASA Dryden Flight Research Center, Edwards AFB, CA 93523.
}

\begin{abstract}
The problem of parameter estimation on hybrid-wing-body aircraft is complicated by the fact that many design candidates for such aircraft involve a large number of aerodynamic control effectors that act in coplanar motion. This adds to the complexity already present in the parameter estimation problem for any aircraft with a closed-loop control system. Decorrelation of flight and simulation data must be performed in order to ascertain individual surface derivatives with any sort of mathematical confidence. Non-standard control surface configurations, such as clamshell surfaces and drag-rudder modes, further complicate the modeling task. In this paper, time-decorrelation techniques are applied to a model structure selected through stepwise regression for simulated and flight-generated lateral-directional parameter estimation data. A virtual effector model that uses mathematical abstractions to describe the multi-axis effects of clamshell surfaces is developed and applied. Comparisons are made between time history reconstructions and observed data in order to assess the accuracy of the regression model. The Cramer-Rao lower bounds of the estimated parameters are used to assess the uncertainty of the regression model relative to alternative models. Stepwise regression was found to be a useful technique for lateraldirectional model design for hybrid-wing-body aircraft, as suggested by available flight data. Based on the results of this study, linear regression parameter estimation methods using abstracted effectors are expected to perform well for hybrid-wing-body aircraft properly equipped for the task.
\end{abstract}

\section{Nomenclature}

$\theta \quad$ pitch angle

$\alpha \quad$ angle of attack

$\bar{q} \quad$ dynamic pressure

$\beta \quad$ angle of sideslip

$\delta a_{j} \quad$ coupled antisymmetric deflection of left and right surfaces $j$, positive for positive roll

Sai deflection of inner clamshell abstracted effector, aileron-like motion

$\delta a o \quad$ deflection of outer clamshell abstracted effector, aileron-like motion

$\delta d i \quad$ deflection of inner clamshell abstracted effector, drag rudder motion

$\delta d o \quad$ deflection of outer clamshell abstracted effector, drag rudder motion

$\delta r$

$\delta s_{j}$ surface deflection of winglet rudders, positive for postive yaw

deflection of surface $j$, positive for trailing edge down

first derivative of angle of sideslip with respect to time

vector of model parameters

roll angle

general case vector of unknowns for error analysis

reference span

*Aerospace Engineer, Aerodynamics and Propulsion Branch, M/S 4840B, AIAA Senior Member.

$\dagger$ Student Trainee (Engineering), Aerodynamics and Propulsion Branch, M/S 4840B, AIAA Student Member.

$\ddagger$ Aerospace Engineer, Controls and Dynamics Branch, M/S4840D, AIAA Member. 
$C_{l} \quad$ coefficient of rolling moment, $\frac{M_{l}}{\bar{q} S b}$

$C_{n} \quad$ coefficient of yawing moment, $\frac{M_{n}}{\bar{q} S b}$

$C_{Y} \quad$ coefficient of side force, $\frac{F_{Y}}{\bar{q} S}$

$E \quad$ expected value operator; or, when used as a subscript, experimental value

$g \quad$ acceleration due to gravity

$I \quad$ identity matrix

$I_{x x} \quad$ roll axis moment of inertia

$I_{x z} \quad$ xz-plane cross-axis moment of inertia

$I_{y y} \quad$ pitch axis moment of inertia

$I_{z z} \quad$ yaw axis moment of inertia

$M \quad$ Fisher information matrix

$m \quad$ aircraft mass

$p \quad$ roll rate

$r$ yaw rate

$r^{2} \quad$ coefficient of determination

$S \quad$ reference area

$s \quad$ model fit error

$V \quad$ magnitude of flight velocity

$X \quad$ regressor matrix

$z \quad$ output vector for the linear regression problem

CRB Cramér-Rao (lower) Bound

DFRC Dryden Flight Research Center

HWB Hybrid-Wing-Body

NASA National Aeronautics and Space Administration

VMS Vehicle Management System

\section{Introduction}

The X-48B Blended Wing Body is an $8.5 \%$ dynamically-scaled, hybrid-wing-body (HWB) aircraft built by Cranfield Aerospace Ltd (United Kingdom); it was the result of a joint partnership between the National Aeronautics and Space Administration (NASA), the Air Force Research Laboratory (AFRL), and The Boeing Company (Chicago, Illinois). The $\mathrm{X}-48 \mathrm{~B}$ and its planned successors are representative of possible future, highly efficient, HWB transport designs that involve integrated configurations using a large number of control effectors. The Environmentally Responsible Aviation project within the NASA Aeronautics Research Mission Directorate intends to use the conclusions drawn from flight research on a series of configurations for the $\mathrm{X}-48 \mathrm{~B}$ to further the development of new, manned, highly efficient transport aircraft. A photograph of the $\mathrm{X}-48 \mathrm{~B}$ in flight at the NASA Dryden Flight Research Center (DFRC) is shown in Fig. 1.

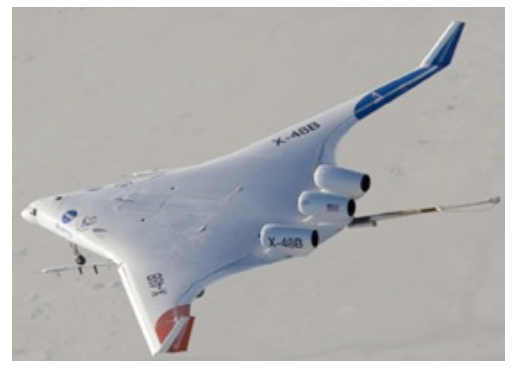

Figure 1. The Boeing X-48B Blended Wing Body, shown in flight near the NASA Dryden Flight Research Center.

Research is being conducted at the NASA Dryden Flight Research Center (DFRC) to study the effectiveness of various parameter estimation methods on HWB aircraft. The problem of parameter estimation on HWB aircraft is complicated by the fact that many design candidates for such aircraft involve a large number of aerodynamic control effectors that act in coplanar motion. This adds to the complexity already present in the parameter estimation problem for any aircraft with a closed-loop control system. Decorrelation of 
flight and simulation data must be performed in order to ascertain individual surface derivatives with any sort of mathematical confidence. Non-standard control surface configurations, such as clamshell surfaces and drag-rudder modes, further complicate the modeling task.

This study applies linear regression parameter estimation techniques to simulation and flight data from the X-48B aircraft, with particular focus on the lateral-directional control surface derivatives. The parameters will be estimated using linear regression methods. A previous paper ${ }^{1}$ that studied the efficacy of standard parameter estimation methods in the longitudinal axis was published in early 2010. The present paper completes that work by analyzing the lateral-directional axes using data from the same flight phase.

\section{Aircraft Description}

The X-48B aircraft incorporates a unique configuration and outer mold line. Instruments relevant to parameter estimation include dual airdata probes to measure airspeed, angle of attack, and angle of sideslip. Additionally, the aircraft is equipped with an Inertial Measurement Unit (IMU) and Global Positioning System (GPS)that provides linear acceleration, angular rotation rates, Euler angles, and position.

The X-48B aircraft can be configured with leading edge slats extended or retracted; however, they cannot be adjusted in flight. Center of gravity can be adjusted on the ground between forward and aft configurations. Allocation of the control surfaces is depicted in Fig. 2 with surface pairs numbered for reference. Additionally, surface 6 and surface 7 are split ailerons, or "clamshell" surfaces; the top and bottom surface can be moved together to produce roll moments or they can be split to produce a yaw moment through differential drag. Rudders are incorporated into the winglets to provide additional yaw control and stability.

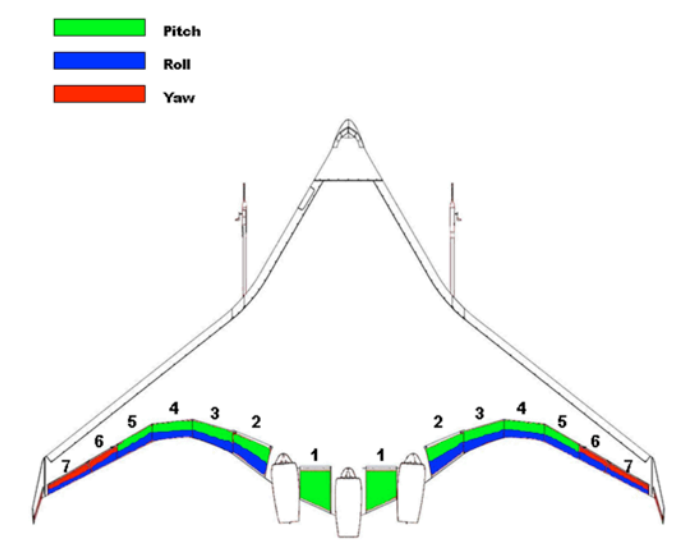

Figure 2. Control surface numbering and axis allocation for the X-48B Blended Wing Body.

Control surface positions are inferred from the measured actuator position and are not measured directly. The control surface actuation on the X-48B aircraft consists of an electro-mechanical servo that moves the control surface through a linkage. Position measurement is taken at the output shaft of the servo; thus, differences between the surface position and actuator position may be due to linkage bending or gear slop. No corrections were made to the control surface data because data or models necessary for corrections were not available.

While these factors complicate any attempt to make absolute quantification of the individual parameters, much work can be done in studying the relative effectiveness of parameter estimation techniques and the associated model structure. Present experimental work by NASA is ongoing to attempt to quantify the uncertainty in the control surface position, as well as the propagated effect into the estimated parameters. This paper applies the models suggested by stepwise regression to simulation and flight data for the X-48B aircraft, analyzes trends in the Cramér-Rao lower bounds, assesses the fit of state-variable time history re- 
constructions from the solved parameters, and verifies the stepwise regression model selection by comparison to possible alternatives.

\section{Method}

Parameter estimation is a subset of the broader field of system identification, wherein the basic task of the engineer is to determine the nature of a system under study through observation and analysis of the outputs generated by a controlled set of inputs. ${ }^{2}$ Parameter estimation assumes that the system in question may be modeled as a parameterized set of equations, of which the coefficients, or parameters, are the objective of the analysis.

The generally accepted standard method of performing maneuvers for parameter estimation are the traditional doublet or triplet inputs. The pilot inputs a simple square wave command of controlled magnitude in a particular axis, and the output dynamics that result from this input are analyzed for a mathematical relationship. In the case of multiple surfaces affecting the same axis, in theory, each surface and associated surface (its opposite pair on the other wing, as well as adjacent surfaces that may provide interference effects) must be tested in all possible combinations for comprehensive model validation. On aircraft with a high number of surfaces, this can be quite time-consuming.

\section{A. Linear Regression for Parameter Estimation}

Linear regression parameter estimation is a technique in which the coefficients of an assumed linear relationship between known inputs and observed outputs are estimated using least-squared fits. More information on linear regression parameter estimation techniques can be found in Klein and Morelli. ${ }^{3}$ Linear regression is often compared to output-error techniques, which actually integrate the equations of motion and compare the state outputs to measured values in order to estimate the parameters. In this sense, linear regression is mathematically simpler and therefore quicker in terms of computational time and complexity. The full derivation of such techniques is beyond the scope of this paper. More information on output-error and maximum likelihood techniques can be found in many available references on the subject. ${ }^{2,4-7}$ Similarly, there are many references ${ }^{8-14}$ that describe prior work to decorrelate control surfaces, including substantial work by Morelli and his colleagues in the field of optimal input design.

The derivation of the Cramér-Rao inequality is given by Maine and Iliff. ${ }^{15}$ The Cramér-Rao Lower Bound represents the lowest magnitude limit for the variance of an estimator with a given bias. Un-modeled dynamics can make the true value of the variance of the estimator much higher. In the simplest case where the variance is assumed to be unbiased and have a normal distribution, the Cramér-Rao bound becomes simply the inverse of the Fisher information matrix, $M\left(\xi_{t}\right)$, which is a metric for measuring the amount of usable information content in a set of data. Choosing input design methods and flight-test techniques that lower the Cramér-Rao bounds is an effective approach to choosing inputs that maximize the usable information content of the flight data.

The derivation of the Cramér-Rao bound assumes that the residuals consist solely of white noise. In practice, this is not the case. As a result, traditionally-computed bounds can be inaccurate. Cramér-Rao bounds presented in this report have been adjusted to account for frequency content in the residuals, using a technique from Klein and Morelli. ${ }^{3}$

\section{B. Data Sources}

Time histories of the aircraft state and motion, air data, control surface position, and other pertinent information for performing parameter estimation problem were obtained from two sources: the Boeing nonlinear simulation, and recorded data from Phase 1 flight tests of the X-48B aircraft at NASA DFRC. For both simulation and flight, force and moment coefficients were constructed from observed air data and flight dynamics using standard aircraft equations of motion.

\section{Maneuver Description}

A series of antisymmetric doublets (equal magnitude in opposite directions, inducing pure roll) was performed for the inner surfaces. For the outer surfaces, clamshell surface maneuvers were divided into pure roll doublets (clamshell halves moving together) and pure yaw doublets (clamshell halves splitting open in pulses). Winglet 
rudder doublets were also performed. For the simulation studies, all surface motions could be performed serially in a single combined maneuver termed a supermanuever, on which regression could be performed. An example is shown in Fig. 3.

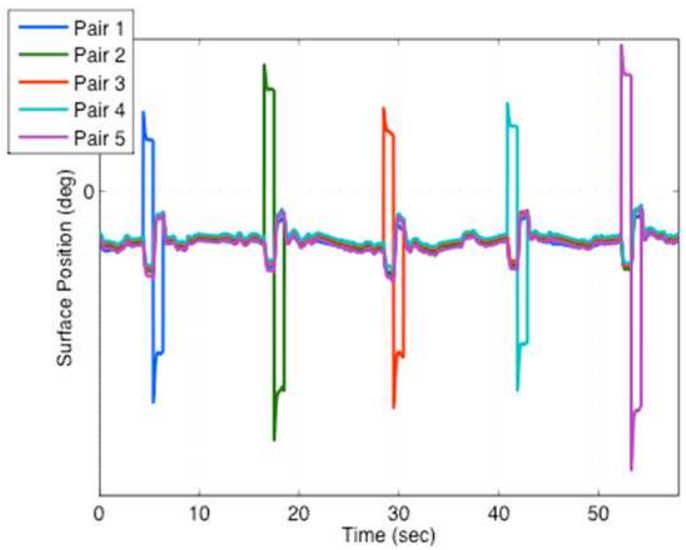

Figure 3. Multi-surface supermaneuver for temporal separation.

It is worthwhile to note that the use of supermanuevers means that every surface excitation in a sequence is present in the dataset for all parameter estimate regression solutions, regardless of which parameters are the focus of the particular model being solved.

\section{Flight-Testing}

Flights 65, 66, and 67 from Phase 1 of the X-48B flight-test program included standard doublet lateraldirectional parameter estimation test points for the present analysis, at angles of attack of $6 \mathrm{deg}, 8 \mathrm{deg}$, and $10 \mathrm{deg}$, respectively. The range of altitudes and corresponding trim speeds for these angles of attack do not vary appreciably enough for a second variable to be considered in defining the flight condition. Individual surface pair maneuvers were available for analysis. Each maneuver was performed three times in flight in order to reduce error and quantify the variance of the dataset.

Phase 1 flight data were gathered as part of Boeing's initial build-up and envelope expansion testing for the X-48B aircraft. Since the test matrix was not designed a priori for the specific parameter estimation research objectives of NASA, researchers on Phase 1 data designed model structures and simulation studies to conform to available flight data. Flight data from individual surface-pair parameter estimation maneuvers, spread out across several different test points, were spliced together into combined supermaneuvers in order to compare to the simulation results.

While smoothing was applied to the transition points to eliminate any discontinuities, two additional factors ameliorated the effect of splicing on the parameter estimation results. First, linear regression, as an equation-error technique, does not rely on integrating the equations of motion as in output-error techniques. This means that there is no need to ensure that integrators are properly reset and that data is exactly aligned at the splicing points for the parameter estimation (though it is required later for time-history reconstruction). Second, the range of flight conditions and possible trim states for the $\mathrm{X}-48 \mathrm{~B}$ aircraft is limited enough that significant disparities in state variables for the same trim angle of attack are unlikely.

Noise present in the flight data was removed using a third-order, two-way Butterworth filter applied with a corner frequency of $5 \mathrm{~Hz}$, because this cutoff appeared to capture the dynamics of interest while excluding the bulk of the measurement and physical noise. Corrections were also made to measured air data as well as translational accelerations to account for the distance from the aircraft center of gravity to the respective measurement points.

\section{Nonlinear Simulation}

The nonlinear simulation of the X-48B aircraft was designed by The Boeing Company and is implemented in Simulink@ (The MathWorks, Natick, Massachusetts). The version of the simulation used for this paper was 4.3.1, using Vehicle Management System (VMS) version 4.3 and aerodynamic model 20091223. 
Though flight is the only aeronautical reality, the advantage of conducting simulation studies is that various real-world effects can be controlled or eliminated, and the isolated effects of various factors on the results can be explored. The simulation results shown in this paper were for supermaneuvers peformed with the flight control laws forced into an open-loop mode. Further, the normal control surface allocator was bypassed in order to insert specific input combinations that may not have been available in the normal control laws.

The simulation manuevers were intially planned for the same angles of attack as the flight data. It became necessary, however, to move the simulation points to take place at trim angles of attack on the half-angle (for example, $6.5 \mathrm{deg}, 7.5 \mathrm{deg}$, et cetera) in order to avoid breakpoints in the aerodynamic model, which were presumably artifiacts from the wind-tunnel test procedure. These breakpoints made finite difference approixations in their vicinity diffcult, which affects related research that would use the same data.

\section{Effector Definitions}

The configuration of the X-48B aircraft allows for several possible definitions of the control effector regressor functions due to the split nature of the clamshell surfaces. Because the clamshell surfaces can move in unison or oppositely, the same clamshell upper and lower surface can behave like a traditional aileron (primarily affecting roll), or split open in a drag-rudder yaw mode.

Deflection of any individual surface will be denoted as $\delta s$; for example, the deflection of the inner surfaces will be represented by $\delta s_{1}$ through $\delta s_{5}$, where the number corresponds to the surface number shown in Fig. 2. When treated individually, the inboard clamshell upper and lower surfaces will be denoted as $\delta s_{6 u}$ and $\delta s_{6 l}$, respectively. The outboard clamshell upper and lower surfaces will be similarly denoted $\delta s_{7 u}$ and $\delta s_{7 l}$. The winglet rudders are located on the wingtips and behave as normal rudders (affecting the directional axis only). The deflection of the winglet rudders is denoted $\delta r$. When an inner surface is coupled into an anti-symmetric pair with its counterpart on the opposite wing of the aircraft, the aileron-like combined deflection can be described by a single abstracted effector, $\delta a$, as shown in Equation 1.

$$
\delta a_{j}=\delta s_{j, l e f t}-\delta s_{j, \text { right }}
$$

Similarly, abstracted effectors were defined to describe the separate lateral and directional effects of the clamshell surfaces, as shown in Equations 2through 5.

$$
\begin{aligned}
& \delta a i=\left(\frac{\delta s_{6 u}+\delta s_{6 l}}{2}\right)_{l e f t}-\left(\frac{\delta s_{6 u}+\delta s_{6 l}}{2}\right)_{\text {right }} \\
& \delta a o=\left(\frac{\delta s_{7 u}+\delta s_{7 l}}{2}\right)_{l e f t}-\left(\frac{\delta s_{7 u}+\delta s_{7 l}}{2}\right)_{\text {right }} \\
& \delta d i=\left(\frac{\delta s_{6 u}-\delta s_{6 l}}{2}\right)_{\text {right }}-\left(\frac{\delta s_{6 u}-\delta s_{6 l}}{2}\right)_{\text {left }} \\
& \delta d o=\left(\frac{\delta s_{7 u}-\delta s_{7 l}}{2}\right)_{\text {right }}-\left(\frac{\delta s_{7 u}-\delta s_{7 l}}{2}\right)_{\text {left }}
\end{aligned}
$$

These definitions create layered mathematical constructs to separately represent clamshell aileron-like movement and clamshell drag-rudder movement for both inboard and outboard clamshell surface pairs. For example, five degrees of $\delta d i$ deflection represents the right-side upper, both open five degrees more away from their average position than the analogous case for the left side, creating a positive yawing moment (to the right) via asymmetric drag.

\section{Model Structure}

The lateral-directional parameter estimation problem seeks to find the linear parameters that, when multiplied by their respective regressor functions, compute accurate values for rolling moment $\left(C_{l}\right)$, yawing moment $\left(C_{n}\right)$, and side force $\left(C_{Y}\right)$. The assumed relationship between the regressor functions and the output force and moment coefficients is the model structure. The general form is shown in Equation 6.

$$
z=X \Theta
$$


where $z$ in this case is the column array of force and moment coefficients, $X$ is the matrix of regressor functions (with individual model equations arranged in rows), and $\Theta$ is the column array of parameters to be estimated. Solving for the parameters yields Equation 7 .

$$
\hat{\Theta}=\left(X^{T} X\right)^{-1} X^{T} z
$$

An example expanded model equation, in this case for coefficient of rolling moment, is shown in Equation 8.

$$
C_{l}=C_{l o}+C_{l \beta} \cdot \beta+C_{l p} \cdot \frac{p b}{2 V}+C_{l r} \cdot \frac{r b}{2 V}+\left[C_{l, \text { surfaces }}\right]
$$

The first four terms on the right-hand side of Equation 8 are quite straighforward: the rolling moment should depend on some bare-airframe bias term (the regressor function for which is simply 1), the angle of sideslip, and the roll and yaw rates of the aircraft. These aerodynamic stability and damping terms will be combined and referred to as $C_{l, a e r o}$ (and like manner for the directional coefficients) for the sake of readability in the remainder of the paper; however, it should be noted that the four described components of $C_{l, a e r o}$ are solved for individually as their own regressor functions.

\section{E. Stepwise Regression}

Stepwise regression ${ }^{3}$ was performed on the data to determine the appropriate model structure. This technique involves the iterative addition and removal of individual terms in the regression equation, and assessing the

effect of each term on the overall model coefficient of determination $\left(r^{2}\right)$ and fit error $(s)$, defined in Equations 9 and 10 , respectively.

$$
\begin{aligned}
r^{2} & =\frac{\hat{\Theta}^{T} X^{T} z-N \bar{z}^{2}}{z^{T} z-N \bar{z}^{2}} \\
s & =\sqrt{\frac{\sum\left(z_{i}-\hat{y}_{i}\right)^{2}}{N-p}}
\end{aligned}
$$

All previously-described regressors were included in the initial pool: the aerodynamic terms, the inner ailerons, the winglet rudders, the outer clamshell surfaces treated singly, and the abstracted effectors constructed from the clamshell surface signals.

Model structures under consideration that treated the clamshell surface halves individually were expected to be inadequate due to the inherently correlated nature of their control allocation scheme. That is, the upper and lower halves of the clamshell surfaces were not permitted to move independently in the flight-test matrix for Phase 1. Such a model might be described by Equation 11, for example, with analogous equations for $C_{n}$ and $C_{Y}$.

$$
C_{l}=C_{l, a e r o}+\left(\sum_{j=2}^{5} C_{l \delta a_{j}} \cdot \delta a_{j}\right)+C_{l \delta s_{6 u}} \cdot 2 \delta s_{6 u}+C_{l \delta s_{l}} \cdot 2 \delta s_{l}+C_{l \delta s_{7 u}} \cdot 2 \delta s_{7 u}+C_{l \delta s_{7 l}} \cdot 2 \delta s_{7 l}+\left(C_{l \delta r} \cdot \delta r\right)
$$

While this type of motion could be studied in the X-48B nonlinear simulation and with the use of the Boeing aerodynamic model, the lack of flight data to support any conclusions would reduce the utility of such a study. The model was applied to a limited set of flight data, however, as a sanity check on the initial reasoning. The abstracted effector definitions were expected to more closely match the actual net input seen by the aircraft.

The remaining question concerns whether to solve the regression problem using combined or split axes. The combined axes model solves the regression for $C_{l}, C_{n}$, and $C_{Y}$ using the same regressors for both lateral and directional coefficients; the split axis models separate the lateral coefficient $\left(C_{l}\right)$ from the two directional coefficients and use different regressors for each axis. The model for combined-axes and abstracted surface effectors utilizes the following model equations, solved using the same regressor set for all axes. The model is mathematically described in Equations 12 through 14. 


$$
\begin{aligned}
& C_{l}=C_{l, a e r o}+\left(\sum_{j=2}^{5} C_{l \delta a_{j}} \cdot \delta a_{j}\right)+\left(C_{l \delta a i} \cdot \delta a i\right)+\left(C_{l \delta a o} \cdot \delta a o\right)+\left(C_{l \delta d i} \cdot \delta d i\right)+\left(C_{l \delta d o} \cdot \delta d o\right)+\left(C_{l \delta r} \cdot \delta r\right) \\
& C_{n}=C_{n, a e r o}+\left(\sum_{j=2}^{5} C_{n \delta a_{j}} \cdot \delta a_{j}\right)+\left(C_{n \delta a i} \cdot \delta a i\right)+\left(C_{n \delta a o} \cdot \delta a o\right)+\left(C_{n \delta d i} \cdot \delta d i\right)+\left(C_{n \delta d o} \cdot \delta d o\right)+\left(C_{n \delta r} \cdot \delta r\right) \\
& C_{Y}=C_{y, a e r o}+\left(\sum_{j=2}^{5} C_{Y \delta a_{j}} \cdot \delta a_{j}\right)+\left(C_{Y \delta a i} \cdot \delta a i\right)+\left(C_{Y \delta a o} \cdot \delta a o\right)+\left(C_{Y \delta d i} \cdot \delta d i\right)+\left(C_{Y \delta d o} \cdot \delta d o\right)+\left(C_{Y \delta r} \cdot \delta r\right)
\end{aligned}
$$

The alternative model for split axes and abstracted surfaces is mathematically described as in Equations 15 through 17.

$$
\begin{gathered}
C_{l}=C_{l, a e r o}+\left(\sum_{j=2}^{5} C_{l \delta a_{j}} \cdot \delta a_{j}\right)+\left(C_{l \delta a i} \cdot \delta a i\right)+\left(C_{l \delta a o} \cdot \delta a o\right) \\
C_{n}=C_{n, \text { aero }}+\left(C_{n \delta d i} \cdot \delta d i\right)+\left(C_{n \delta d o} \cdot \delta d o\right)+\left(C_{n \delta r} \cdot \delta r\right) \\
C_{Y}=C_{Y, \text { aero }}+\left(C_{Y \delta d i} \cdot \delta d i\right)+\left(C_{Y \delta d o} \cdot \delta d o\right)+\left(C_{Y \delta r} \cdot \delta r\right)
\end{gathered}
$$

The split axis model was not expected to perform as well as the combined axis model; limited comparisons were made of the Cramér-Rao bounds for parameters common to both models to verify this. In this case the lateral and directional coefficients are calculated in two regression solutions, using separate regressors for the lateral and directional axes. The results are then merged. A summary of the stepwise regression results for a supermaneuver constructed from noise-filtered flight data taken at 6.5 deg angle of attack is shown in Table 1. Flight data were chosen, as noiseless simulation data have been shown to be fairly immune to correlation. ${ }^{16}$

\begin{tabular}{|c|c|c|c|}
\hline Model & Structure & $r^{2}$ & $s$ \\
\hline \hline$C_{Y}$ & Combined axes / abstracted effectors & $\mathbf{9 5 . 0 3 \%}$ & $\mathbf{1 7 . 4 2 \%}$ \\
\hline & Combined axes / individual surfaces & $94.06 \%$ & $17.54 \%$ \\
\hline & Split axes / abstracted effectors & $93.20 \%$ & $20.52 \%$ \\
\hline$C_{l}$ & Combined axes / abstracted effectors & $\mathbf{9 0 . 2 2 \%}$ & $\mathbf{3 1 . 2 1 \%}$ \\
\hline & Combined axes / individual surfaces & $89.59 \%$ & $32.21 \%$ \\
\hline & Split axes / abstracted effectors & $76.90 \%$ & $48.05 \%$ \\
\hline$C_{n}$ & Combined axes / abstracted effectors & $\mathbf{9 3 . 7 9 \%}$ & $\mathbf{2 4 . 7 7 \%}$ \\
\hline & Combined axes / individual surfaces & $93.23 \%$ & $25.87 \%$ \\
\hline & Split axes / abstracted effectors & $90.67 \%$ & $30.17 \%$ \\
\hline
\end{tabular}

Table 1. Average coefficient of determination and fit error from stepwise regression for representative model structures, performed on three repetitions of flight data at $10 \mathrm{deg}$ trim angle of attack, with the chosen model highlighted in bold.

From Table 1 it can be seen that the model using combined-axis regressors and the abstracted effector definitions performs very well, with high coefficients of determination and low fit errors for all three desired force and moment coefficients. The model with individual effectors also performs surprisingly well, considering the expected cross-correlation between surface halves. However, the abstracted effector model performs slightly better. Using stepwise regression, the best approach to modeling the yaw effector regressors was found to be one of combined axes and abstracted effectors, as described by Equations 12 through 14. This was the model chosen for application and is used throughout the rest of this paper except where noted for sanity check comparisons to the alternative models. 


\section{Results and Discussion}

Due to the proprietary nature of the performance data of the X-48B aircraft, all plots in this section will be provided without quantification on the ordinate axis. The flight-data results are provided with error bars, which represent the Cramér-Rao Lower Bounds for the respective parameter estimate data points.

Many of the provided results depict trends for simulation and flight that do not agree with each other in slope or other characteristics. While the simulation and flight results will be compared as a sanity check to ensure that the parameter estimation results for flight do not wildly deviate from expected values, it is not the objective of this paper to assess the accuracy of the Boeing nonlinear simulation or aerodynamic model with respect to flight. The objective is rather to assess the quality of the lateral-directional model suggested by stepwise regression on multiple HWB aircraft datasets. The best measure of the effectiveness of the model lies in its ability to more accurately reconstruct observed state-variable time histories. In doing so, the best model should also perform with lower Cramér-Rao bounds than do other models for the same dataset. The model is here applied to two different datasets for the X-48B aircraft: simulation and flight. Any disparity between the two sets of results suggests that the aerodynamic model inadequately captures certain aerodynamics of the flight vehicle; it does not affect the analysis of the parameter estimation model.

\section{A. Inner Surfaces}

For the inner surfaces, one would expect relatively clean results for the rolling moment derivatives $C_{l \delta a_{2}}$ through $C_{l \delta a_{5}}$, as the maneuvers performed for the lateral-directional parameter estimation were antisymmetric roll doublets. However, this is not strongly the case. Figure 4 shows coefficient of rolling moment with respect to surface pair 4 differential deflection, plotted against angle of attack for simulation and flight data.

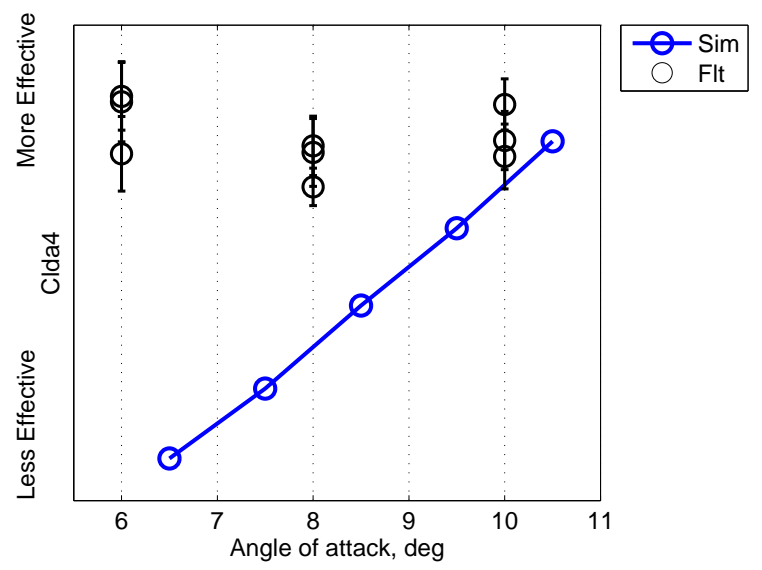

Figure 4. Derivative of rolling moment coefficient with respect to $\delta a 4$, representative of rolling moment derivatives with respect to inner surface roll input.

Figure 4 shows flight data parameter estimates for which two repetitions fall tightly together for each angle of attack. One repetition falls outside the Cramér-Rao bounds for at least one of the two other points. While ideally, for a perfect model, every independent estimate of a particular parameter should fall within the bounds of every other independent estimate, this model is reasonably consistent overall. The simulation parameter estimation does not accurately capture the slope of the trend seen in flight in any of the $C_{l \delta a}$ plots. In these respects, the Fig. 4 plot is representative of all four inner rolling moment surface derivatives.

It is surprising to note that the simulation tracks closer to flight for the off-axis derivatives, $C_{n \delta a}$, as shown by the representative plot in Fig. 5. For these derivatives, the flight data parameter estimates are clustered within Cramér-Rao bounds for lower angles of attack, with the tightness of the cluster deteriorating slightly but noticeably with angle of attack.

The model produces tight precision for off-axis side force $\left(C_{y \delta a}\right)$ derivatives, as shown in Fig. 6. For these derivatives, the spread does not worsen with angle of attack, although the simulation exhibits a notably opposite trendline. The aerodynamic model and corresponding control allocation scheme are not, however, designed to consider using the inner surfaces as yaw effectors. 


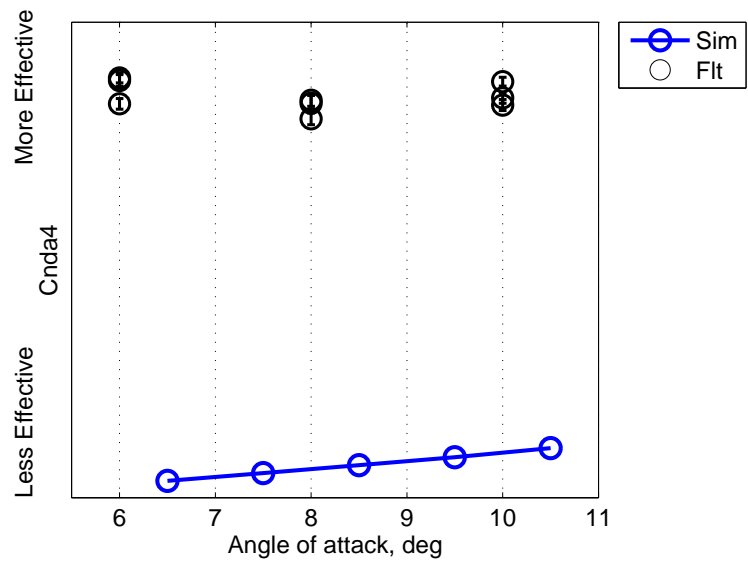

Figure 5. Derivative of yawing moment coefficient with respect to $\delta a 4$, representative of inner yawing moment derivatives with respect to roll input.

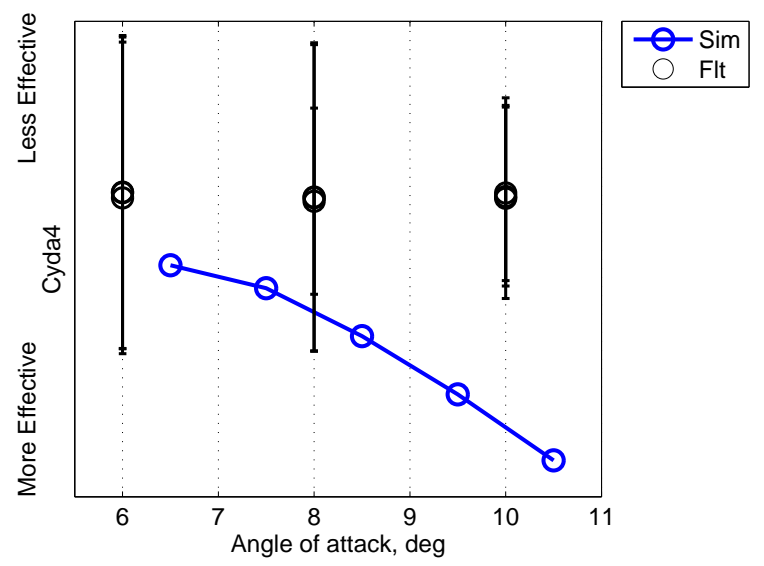

Figure 6. Derivative of side force coefficient with respect to $\delta a 4$, representative of inner side force derivatives with respect to roll input. 
For all inner surface derivatives, the flight parameter estimates for multiple repetitions at each angle of attack are generally clustered within each other's calculated Cramér-Rao lower bounds. From these results it may be concluded that the model is consistent. The simulation results in the vicinity of the flight data results provide a sanity check on the parameters as well, showing that in addition to consistency, the model is calculating parameters that are within reasonable distance of aerodynamic model predictions. The differences in the trends between simulation and flight indicate unmodeled physics in the aicraft aerodynamic model; however, such differences do not affect the conclusions drawn on the parameter estimation model, as this model was not the one used to simulate the aircraft.

\section{B. Outer Surfaces}

The outer surfaces show on-par or better precision as compared to the standard input definitions for the inner surfaces. Figure 7 shows flight data parameter estimates for rolling moment with respect to inner clamshell aileron-like motion. The data points are well-clustered and within each other's Cramér-Rao bounds for all angles of attack. The simulation shows a similar trend, but offset with a bias. The abstracted clamshell aileron effector does well for the off-axis derivatives as well, predicting yawing moment coefficient in a consistent manner as shown in Figure 8. In this latter case, the simulation is notably better at tracking the flight results.

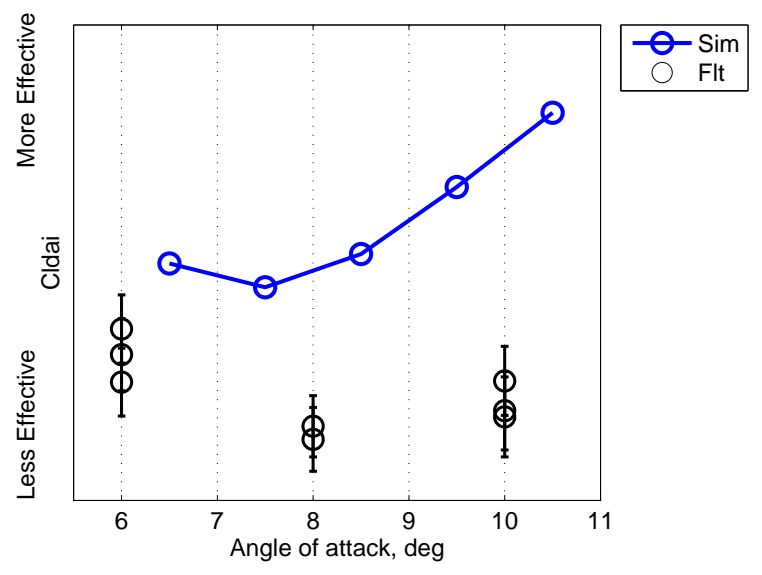

Figure 7. Derivative of rolling moment coefficient with respect to $\delta a i$ (clamshell aileron-like motion).

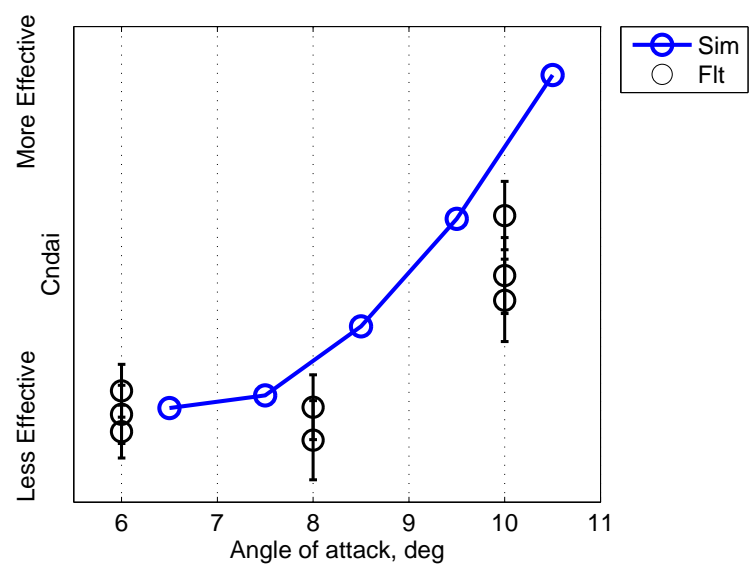

Figure 8. Derivative of yawing moment coefficient with respect to $\delta a i$ (clamshell aileron-like motion).

Figure 9 shows the yaw axis performance of the abstracted effectors. Yawing moment with respect to outer clamshell drag-rudder motion is predicted very consistently, with the exception of the outlier visible 
in one repetition at 8 degrees trim angle of attack. Figure 10 again shows high consistency, this time for the off-axis rolling moment derivative with respect to a clamshell yawing input. The same repetition at 8 deg trim angle of attack appears as an outlier.

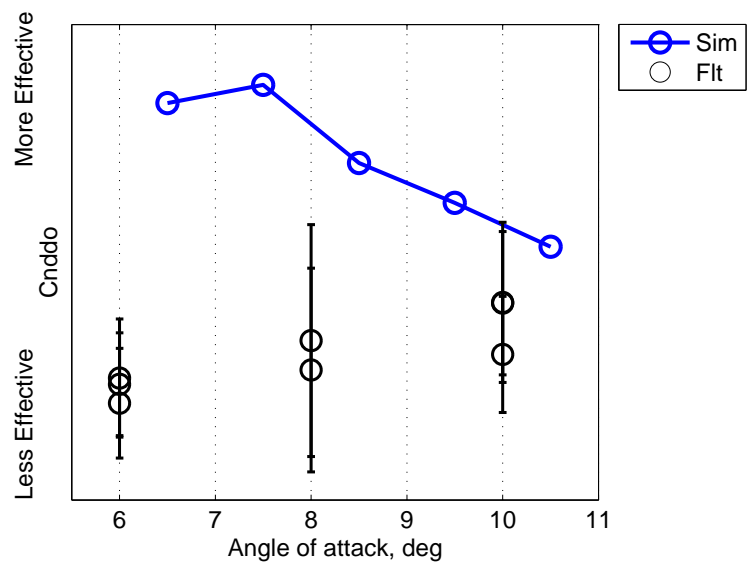

Figure 9. Derivative of yawing moment coefficient with respect to $\delta d o$ (clamshell differential drag-rudder).

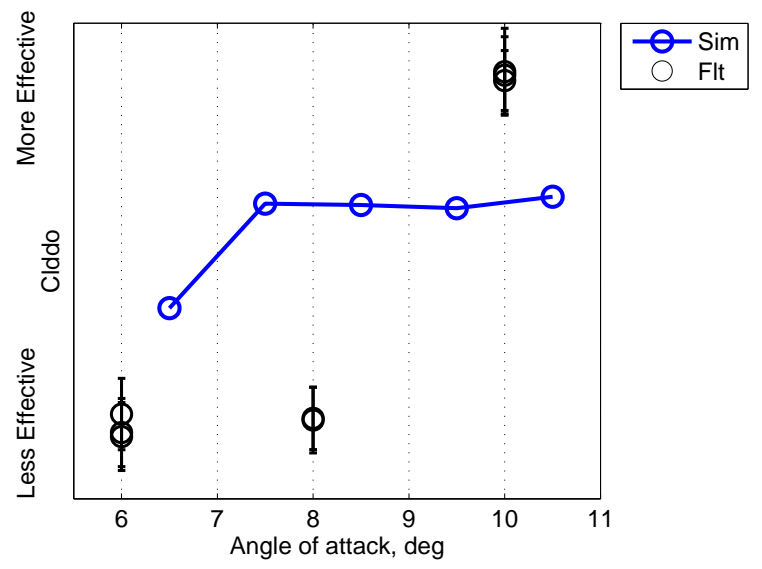

Figure 10. Derivative of rolling moment coefficient with respect to $\delta d o$ (clamshell differential drag-rudder).

\section{Model Quality}

The primary performance metric for model accuracy is the fit of the time history reconstructions. That is, does the model successfully predict the actual system behavior when subjected to the same inputs? Further, while doing so, does the model exhibit lower Cramér-Rao lower bounds than alternative models?

\section{State-Variable Time History Reconstructions}

Time history reconstructions of the aircraft state variables as calculated by the various models can be compared to the observed data in simulation and flight in order to quantify the model error. Using the solved set of parameters, the force and moment coefficients can be calculated for each time frame and inserted into the aircraft equations of motion. As per Klein and Morelli, ${ }^{3}$ the lateral-directional equations of motion for small angles of beta using measured values are shown in Equations 18 through 20.

$$
\dot{\beta}=\frac{\bar{q}_{E} S}{m V_{E}} C_{Y}+p \sin \alpha_{E}-r \cos \alpha_{E}+\frac{g}{V_{E}} \sin \phi_{E} \cos \theta_{E}
$$




$$
\begin{aligned}
& \dot{p}-\frac{I_{x z}}{I_{x x}} \dot{r}=\frac{\bar{q}_{E} S b}{I_{x x}} C_{l}-\frac{\left(I_{z z}-I_{y y}\right)}{I_{x x}} q_{E} r+\frac{I_{x z}}{I_{z z}} q_{E} p \\
& \dot{r}-\frac{I_{x z}}{I_{z z}} \dot{p}=\frac{\bar{q}_{E} S b}{I_{z z}} C_{n}-\frac{\left(I_{y y}-I_{x x}\right)}{I_{z z}} q_{E} p+\frac{I_{x z}}{I_{z z}} q_{E} r
\end{aligned}
$$

In these equation, the subscript $E$ indicates experimental values. The term representing the contribution of the engine rotation to yaw rate is omitted from Equation 20. Similar equations may be found in Maine and Iliff ${ }^{6}$ for example, or any number of papers and textbooks on aircraft dynamics. These equations of motion are then integrated to arrive at state variables, which are then compared to observation.

Figures 11 and 12 show time history reconstructions of angle of sideslip and the coefficient of side force for both simulation and flight data, respectively. The time slice is for a outer-surface drag-rudder $(\delta d o)$ motion, with the simulation data calculated at 6.5 degrees trim angle of attack, and the flight data observed at 6 degrees angle of attack.

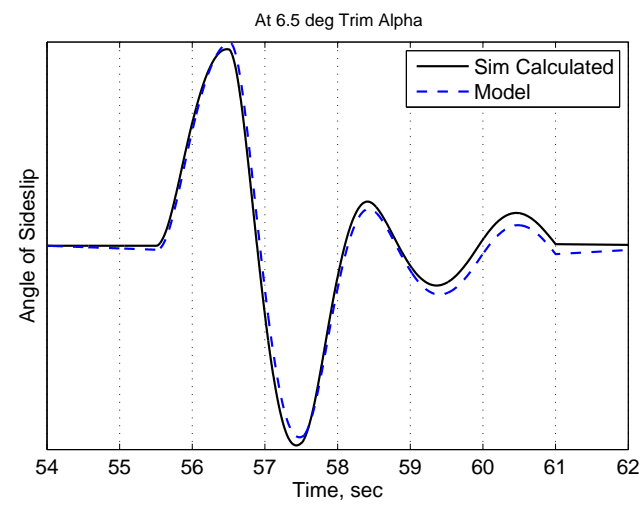

(a) Angle of sideslip, $\alpha_{\text {trim }}=6.5 \mathrm{deg}$.

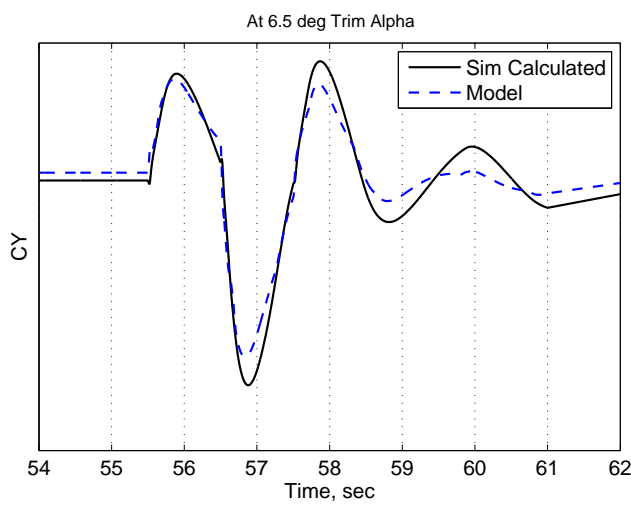

(b) Coefficient of side force, $\alpha_{\text {trim }}=6.5 \mathrm{deg}$.

Figure 11. Simulation time history reconstructions of angle of sideslip and coefficient of side force.

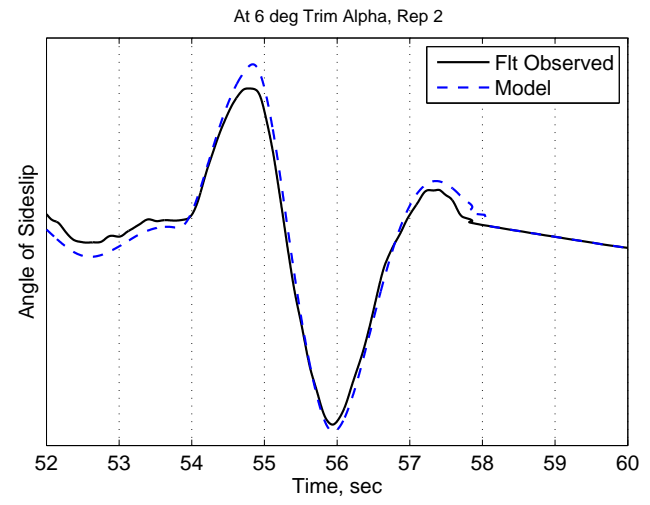

(a) Angle of sideslip, $\alpha_{\text {trim }}=6 \mathrm{deg}$.

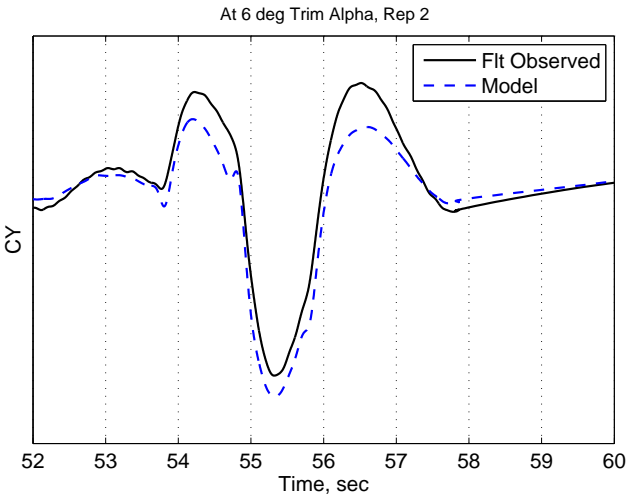

(b) Coefficient of side force, $\alpha_{\text {trim }}=6 \mathrm{deg}$.

Figure 12. Flight-data time history reconstructions of angle of sideslip and coefficient of side force.

It can be seen that the chosen parameter estimation model accurately reconstructs the simulation data, which was calculated for an open-loop system. The end of the maneuver in Fig. 11(b) is less well constructed, and it can be seen that the reconstructed angle of sideslip in Fig. 11(a) begins to deviate at the same point (approximately 58.5 seconds). The fit would actually deviate more if not for the fact that the integrator is reset at the end of the manuever, forcing the reconstruction back to the observed data before beginning the next doublet. This was done to accomodate the splicing method used to assemble the supermaneuvers. 
In Fig. 12(a), slight errors in the flight angle of sideslip reconstruction at the peaks and troughs of the signal can be traced to the same misses in the coefficient, see Fig.12(b). The fit for both is noticeably less accurate for flight data when compared to simulation, which is to be expected, since the open-loop simulation is an idealized environment. The ideal nature of the simulation should lead to nearly perfect model reconstruction; the inaccuracies in Fig. 11(b) are not present in simulation reconstructions of the other lateral-directional coefficients 15(a). These plots show reconstructions for simulation data, whereas the regression model structure was selected using flight data. While the simulation has appeared to be different from the flight aircraft in several instances, the best overall model structure should not be significantly different. The loose fit in Fig. 12(b) does indicates that the side force model is not entirely complete, and could warrant further attention.

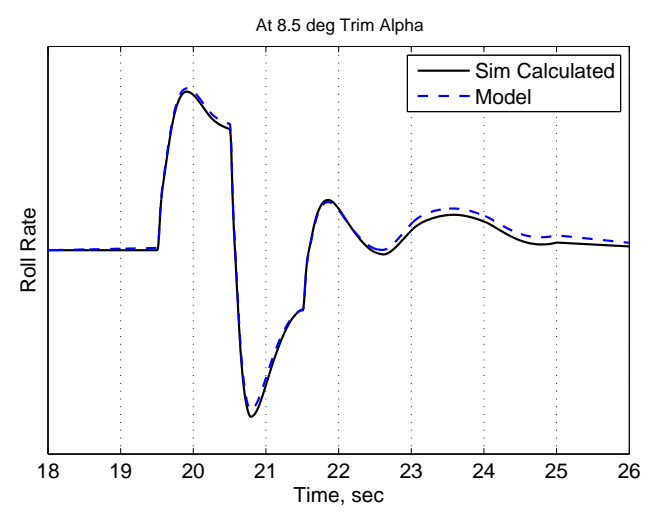

(a) Roll rate, $\alpha_{\text {trim }}=8.5 \mathrm{deg}$.

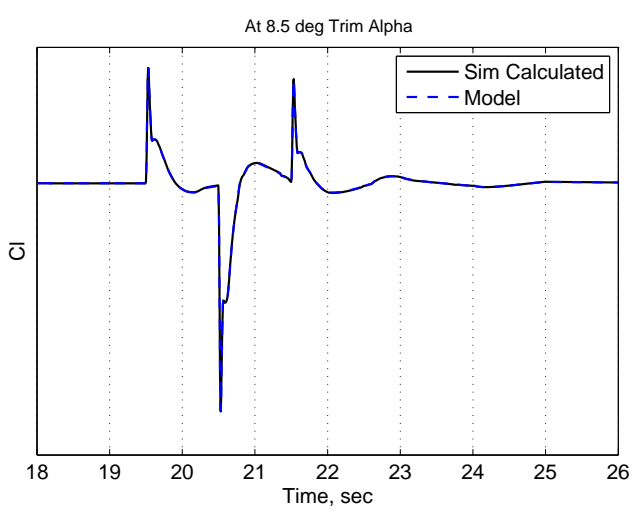

(b) Coefficient of rolling moment, $\alpha_{\text {trim }}=8.5 \mathrm{deg}$.

Figure 13. Simulation time history reconstructions of roll rate and coefficient of rolling moment.

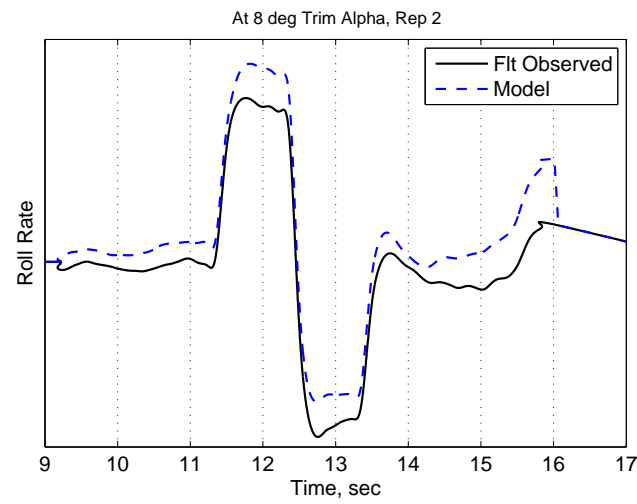

(a) Roll rate, $\alpha_{\text {trim }}=8 \mathrm{deg}$.

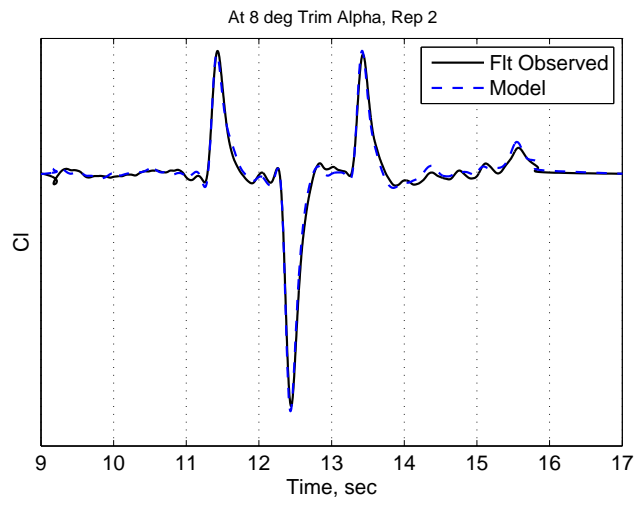

(b) Coefficient of rolling moment, $\alpha_{\text {trim }}=8 \mathrm{deg}$.

Figure 14. Flight-data time history reconstructions of roll rate and coefficient of rolling moment.

Figures 13 and 14 show reconstructions of roll rate and coefficient of rolling moment for simulation and flight, respectively. The simulation reconstructions for both are very accurate. The flight reconstruction of $C_{l}$ is quite accurate as well, though the reconstruction of roll rate (Fig. 14(a)), while capturing the appropriate trends and timing, has a noticeably poorer fit. Since the coefficient appears to be accurately reconstructed in Fig. 14(b) and all other terms in Equation 19 are measured quantities from flight, it is presumed that uncertainty in the mass properties of the aircraft could be causing these fit errors. Swing testing is underway at NASA DFRC to attempt to quantify uncertainty related to the mass properties model of the X-48B aircraft, and to assess the impact on the parameter estimation process.

Figures 15 and 16 show reconstructions of yaw rate and coefficient of yawing moment for simulation and flight, respectively. Conclusions similar to those just described for the roll axis may be drawn. 


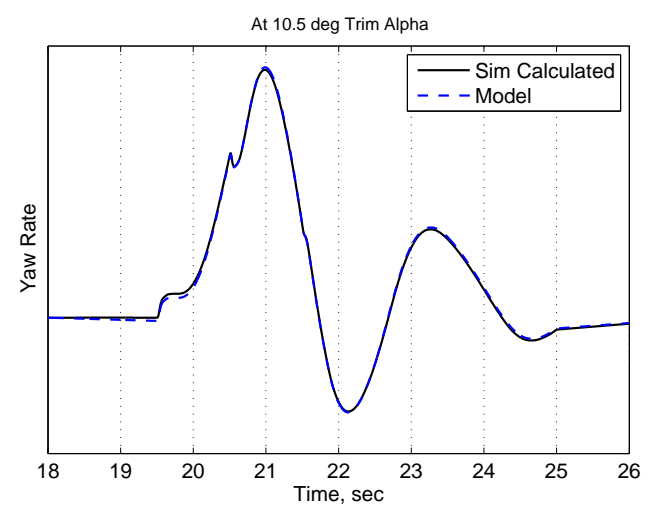

(a) Yaw rate, $\alpha_{\text {trim }}=10.5 \mathrm{deg}$.

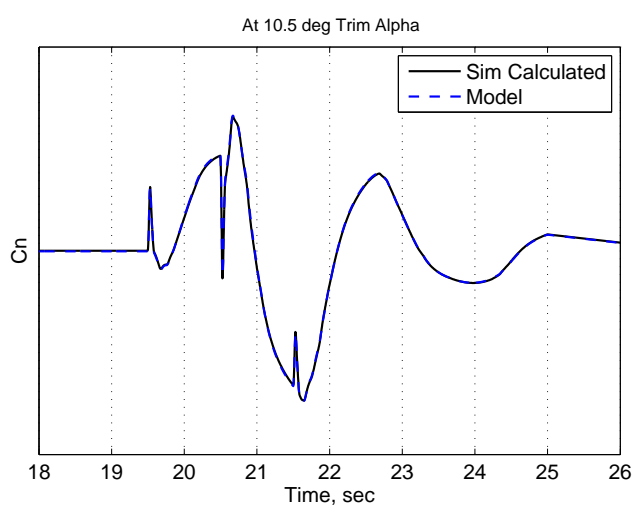

(b) Coefficient of yawing moment, $\alpha_{\text {trim }}=10.5 \mathrm{deg}$.

Figure 15. Simulation time history reconstructions of yaw rate and coefficient of yawing moment.

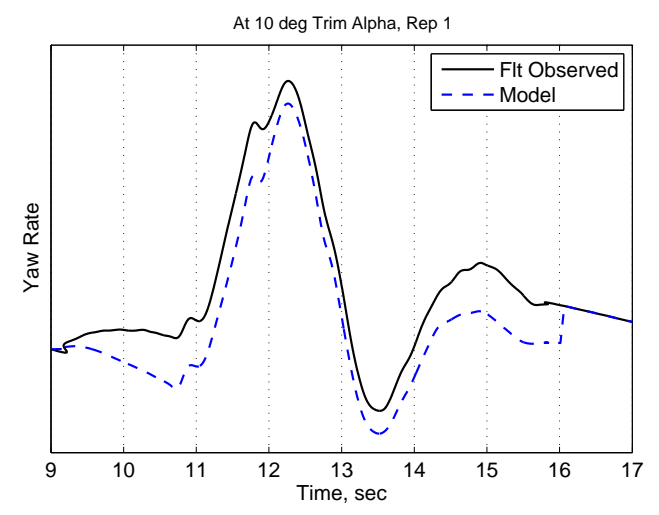

(a) Yaw rate, $\alpha_{\text {trim }}=10 \mathrm{deg}$.

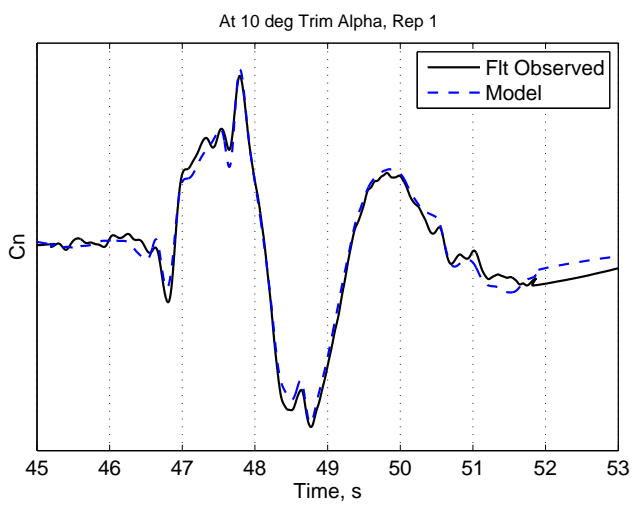

(b) Coefficient of yawing moment, $\alpha_{\text {trim }}=10 \mathrm{deg}$.

Figure 16. Flight-data time history reconstructions of yaw rate and coefficient of yawing moment. 


\section{Cramér-Rao Lower Bounds}

Figure 17 shows Cramér-Rao bounds for the flight data paramter estimates on the rolling moment coefficient with respect to a roll input from an abstracted surface. The primary model (combined axes regressors with abstracted effector models) is shown along with the alternative models for split axes described previously. Both models are shown to exhibit Cramér-Rao bounds consistent with expected levels. The selected (combined axis) model performs better than the split axis model, confirming the intial model selection performed eariler via stepwise regression. The results shown in Fig. 17 are representative of the general results.

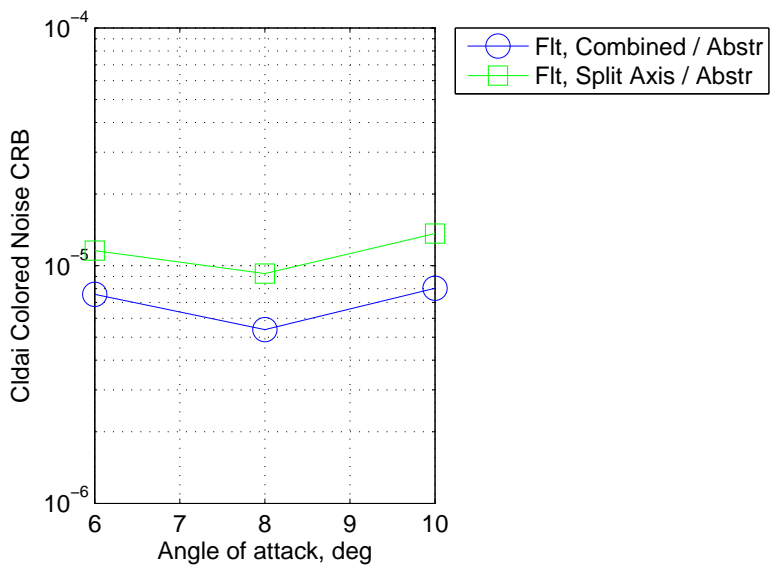

Figure 17. Cramér-Rao lower bounds for $C_{l \delta a i}$ estimates using alternative model structures (combined axis / abstracted effectors being the primary model).

\section{Concluding Remarks}

Stepwise regression was used to construct a linear regression model of the X-48B lateral-directional dynamics using combined-axis regressors and abstracted effectors for the clamshell surfaces. From the stepwise regression results, two major conclusions may be drawn:

1. Accounting for cross-axis dynamics improves model fidelity despite the increased complexity of the regresion equation. In other words, attempting to simplify the model by splitting the dynamics into pure-axis derivatives actually detracts from model fidelity.

2. Defining mathematical abstractions for complex surfaces that more accurately capture the effective total input seen by the system can result in higher model fidelity than treating the physical surface motion literally.

The metrics for model performance were that increased accuracy of the state-variable time history reconstructions connoted a higher-fidelity model, and that lower Cramér-Rao bounds connoted higher confidence in the respective estimates. Overall, these metrics were met for both simulation and flight data. Linear regression parameter estimation methods using abstracted effectors are therefore expected to perform well (delivering consistent, accurate, and high-confidence estimates) for hybrid-wing-body aircraft properly equipped for the task. Further, the Cramér-Rao bounds for the selected model were found to be generally lower than an alternative model for representative parameters. Thus stepwise regression was found to be a useful technique for lateral-directional model design for hybrid-wing-body aircraft, as validated by available flight data.

\section{Future Work}

Future parameter estimation studies of advanced single-surface and optimal input excitations on the $\mathrm{X}-48 \mathrm{~B}$ aircraft will expand on the present research and that of the longitudinal parameters studied previously. ${ }^{1}$ Future studies will also be compared to the baseline estimates using the standard methods found in the studies currently at hand. From this research, the team intends to draw conclusions on the relative effectiveness and accuracy of various standard and advanced parameter estimation methods on hybrid-wing- 
body aircraft. Analysis of advanced parameter estimation inputs, which flew in late calendar year 2010 on the $\mathrm{X}-48 \mathrm{~B}$ aircraft, will continue in this ongoing research. These more advanced inputs include attempting multiaxis identifiabilty using single-surface manuevers, as well as optimal input Walsh Function ${ }^{10}$ excitation for decorrelation.

\section{References}

\footnotetext{
${ }^{1}$ Taylor, B.R. and Ratnayake, N.A., "Simulation and Flight Evaluation of a Parameter Estimation Input Design Method for Hybrid-Wing-Body Aircraft," AIAA 2010-7949, 2010.

${ }^{2}$ Maine, R. E. and Iliff, K. W., "Identification of Dynamic Systems-Theory and Formulation," NASA RP-1138, 1985.

${ }^{3}$ Klein, V. and Morelli, E.A., Aircraft System Identification: Theory and Practice, AIAA Education Series, 2006.

${ }^{4}$ Steers, S. T. and Iliff, K. W., "Effects of Time-Shifted Data on Flight-Determined Stability and Control Derivatives," NASA TN D-7830, 1975.

${ }^{5}$ Iliff, K. W. and Maine, R. E. and Montgomery, T. D., "Important Factors in the Maximum Likelihood Analysis of Flight Test Maneuvers," NASA TP-1459, 1979.

${ }^{6}$ Maine, R.E. and Iliff, K.W., "Application of Parameter Estimation to Aircraft Stability and Control - The Output Error Approach," NASA RP-1168, 1986.

${ }^{7}$ Trujillo, B. M., "Determination of Lift and Drag Characteristics of Space Shuttle Orbiter Using Maximum Likelihood Estimation Technique," AIAA 86-2225, 1986.

${ }^{8}$ Neto, N.S.B. and Hemerly, E.M. and Goes, L.C.S., "Aircraft Parameter Estimation Experiment Design Considering Measurement Colored Residuals," Journal of Aircraft, Vol. 46, No. 6, 2009, pp. $1857-1865$.

${ }^{9}$ Morelli, E. A., "F-18 High Alpha Research Vehicle (HARV) Parameter Identification Flight Test Maneuvers for Optimal Input Design Validation and Lateral Control Effectiveness," NASA CR-198248, 1995.

${ }^{10}$ Morelli, E.A., "Flight Test Validation of Optimal Input Design and Comparison to Conventional Inputs," AIAA-97-3711, 1997.

${ }^{11}$ Morelli, E. A., "Flight-Test Experiment Design for Characterizing Stability and Control of Hypersonic Vehicles," Journal of Guidance, Control, and Dynamics, Vol. 32, No. 3, 2009, pp. 949-959.

${ }^{12}$ Morelli, E. A., "Multiple Input Design for Real-Time Parameter Estimation in the Frequency Domain," 13th IFAC Symposium on System Identification, 2003.

${ }^{13}$ Morelli, E. A., "Practical Input Optimization for Aircraft Parameter Estimation Experiments," NASA CR$191462,1993$.

${ }^{14}$ Morelli, E. A. and Klein, V., "Optimal Input Design for Aircraft Parameter Estimation using Dynamic Programming Principles," AIAA 90-2801-CP, 1990.

${ }^{15}$ Maine, R.E. and Iliff, K.W., "Theory and Practice of Estimating the Accuracy of Dynamic Flight-Determined Coefficients," NASA RP-1077, 1981.

${ }^{16}$ Morelli, E.A., "Practical Aspects of the Equation-Error Method for Aircraft Parameter Estimation," 2006.
} 


\title{
Lateral-Directional Parameter Estimation on the X-48B Aircraft Using an Abstracted, Multi-Objective Effector Model
}

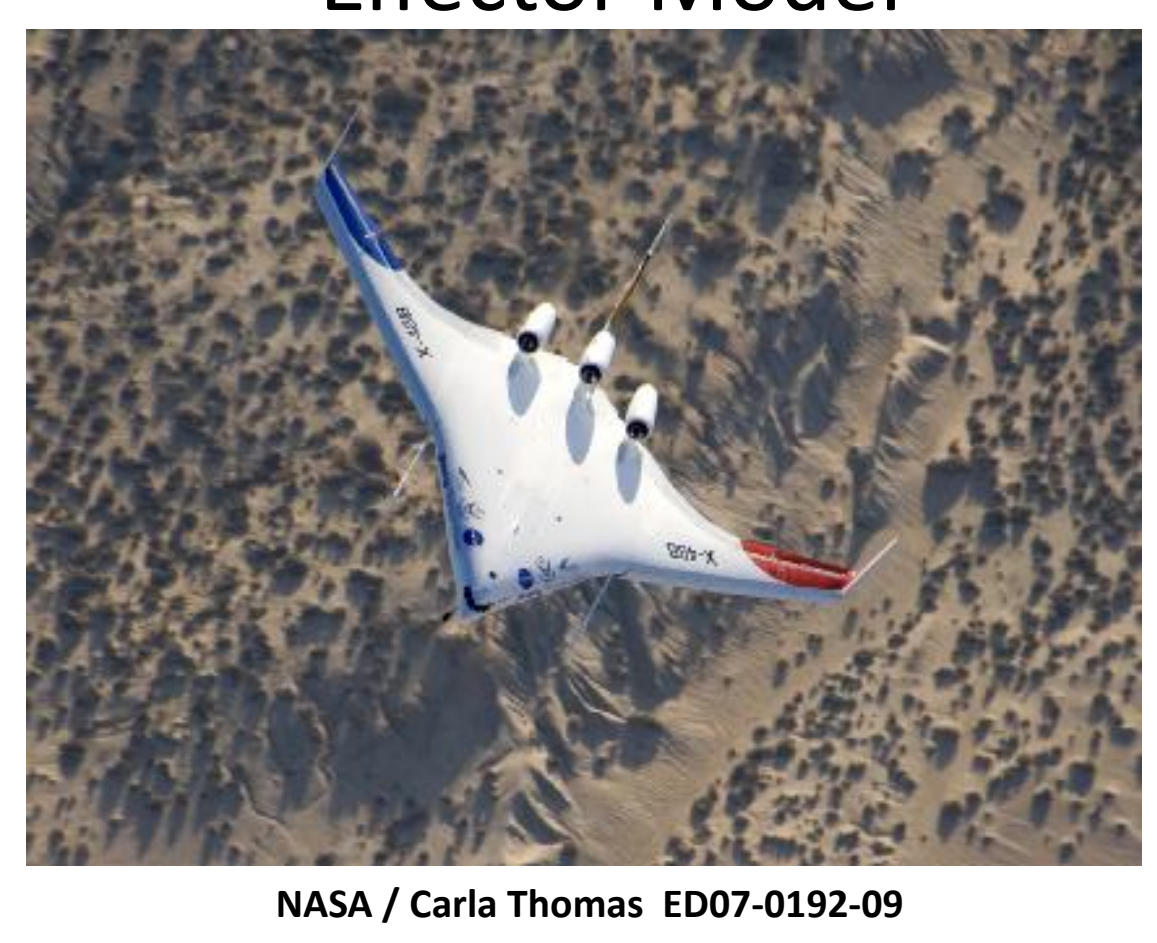

\author{
Nalin A. Ratnayake \\ Erin R. Waggoner \\ Brian R. Taylor
}

NASA Dryden Flight Research Center 


\section{Problem Statement}

- Hybrid-Wing Body aircraft such as the X-48B BWB potentially the future of highly efficient air transport

- New systems will possibly require new ways of approaching the system identification problem

- Math is the same, but...

- New configurations = new issues

- Identifiability!

- Many co-planar surfaces

- Complex outer surface motion (clamshell)

- This paper: Lateral-Directional estimation using "baseline" methods for the X-48B

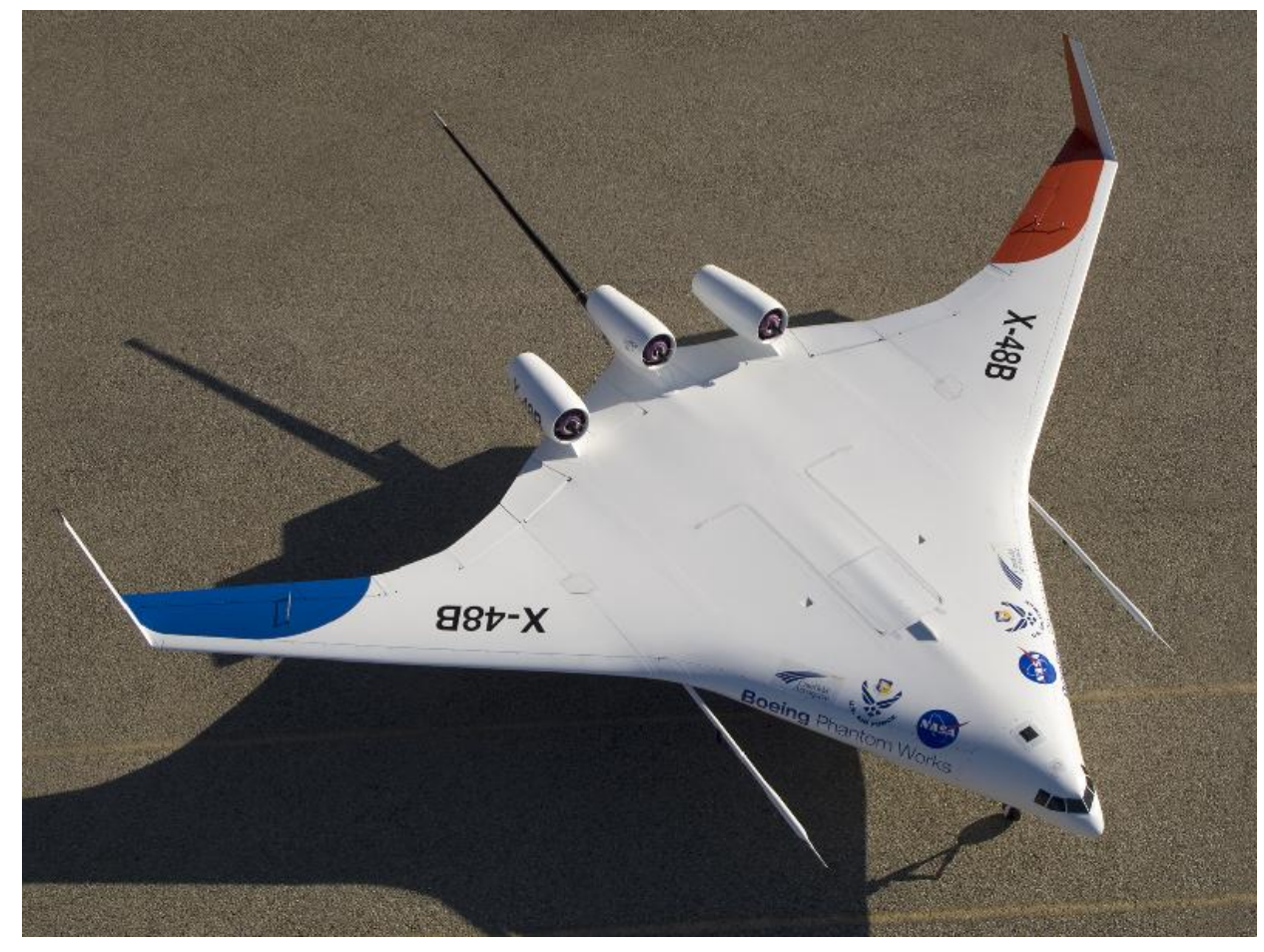

NASA / Tony Landis (ED06-0198-37) 


\section{Surface Definitions}

- Surfaces 1: elevator motion

- Surfaces 2-5: elevon motion

- Surfaces 6/8 and 7/9: clamshell motion

- Aileron-like for roll

- Split motion for drag rudder mode

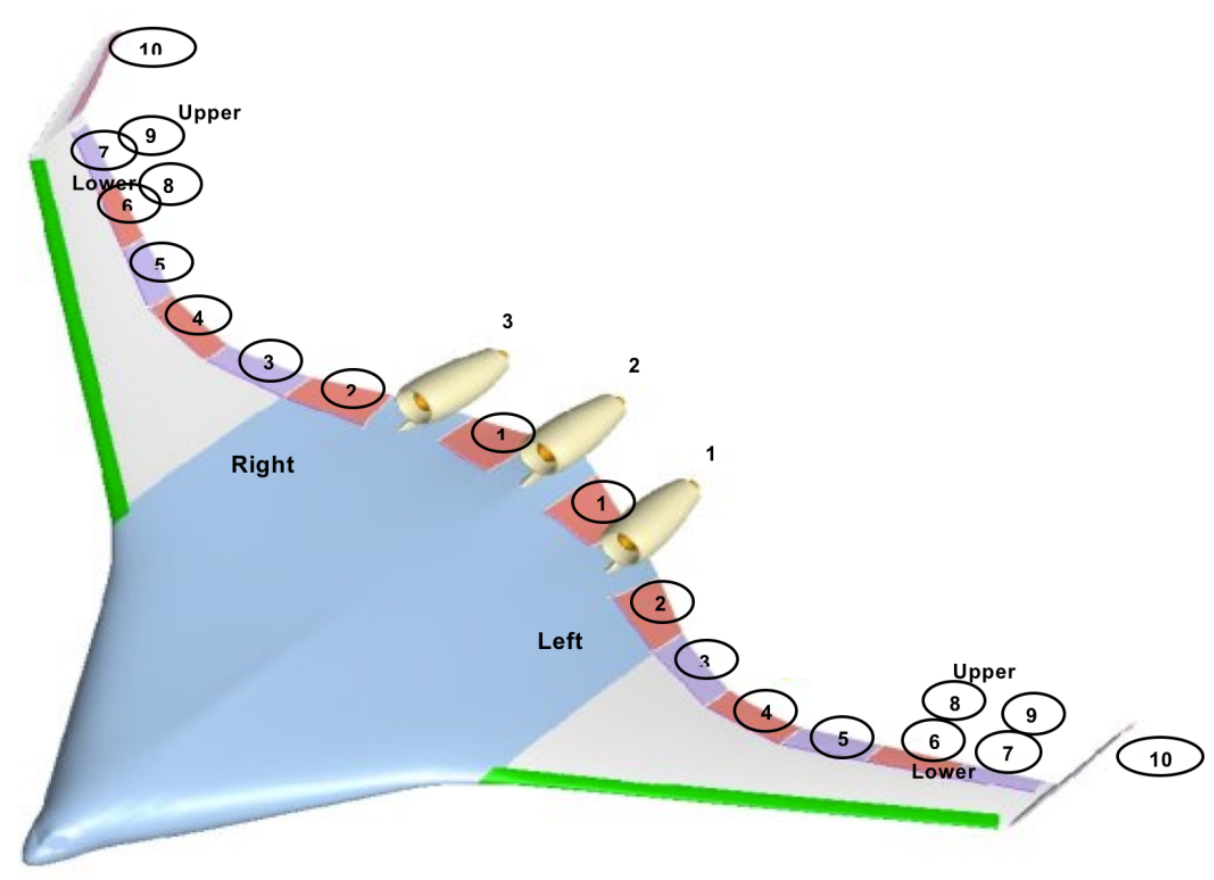

- Winglet rudders 


\section{Surface Definitions}

$$
\begin{gathered}
\delta a_{j}=\delta s_{j, \text { left }}-\delta s_{j, \text { right }} \\
\delta a i=\left(\frac{\delta s_{6 u}+\delta s_{6 l}}{2}\right)_{l e f t}-\left(\frac{\delta s_{6 u}+\delta s_{6 l}}{2}\right)_{\text {right }} \\
\delta a o=\left(\frac{\delta s_{7 u}+\delta s_{7 l}}{2}\right)_{l e f t}-\left(\frac{\delta s_{7 u}+\delta s_{7 l}}{2}\right)_{\text {right }} \\
\delta d i=\left(\frac{\delta s_{6 u}-\delta s_{6 l}}{2}\right)_{\text {right }}-\left(\frac{\delta s_{6 u}-\delta s_{6 l}}{2}\right)_{l e f t} \\
\delta d o=\left(\frac{\delta s_{7 u}-\delta s_{7 l}}{2}\right)_{\text {right }}-\left(\frac{\delta s_{7 u}-\delta s_{7 l}}{2}\right)_{l e f t}
\end{gathered}
$$

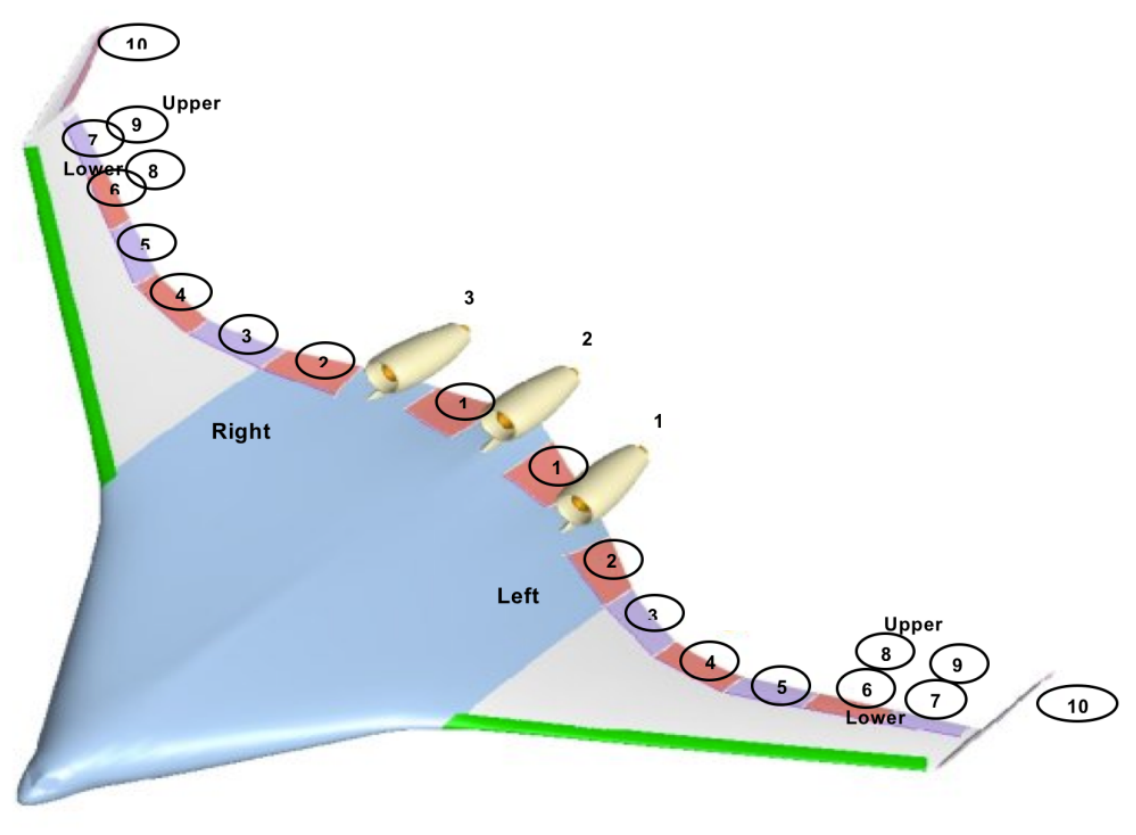




\section{Supermaneuver ${ }^{\circledR \mathrm{TM}} *$}

- Individual doublets are spliced together into a single data file for combined analysis

- 2 sec time frame skip to reset integrators
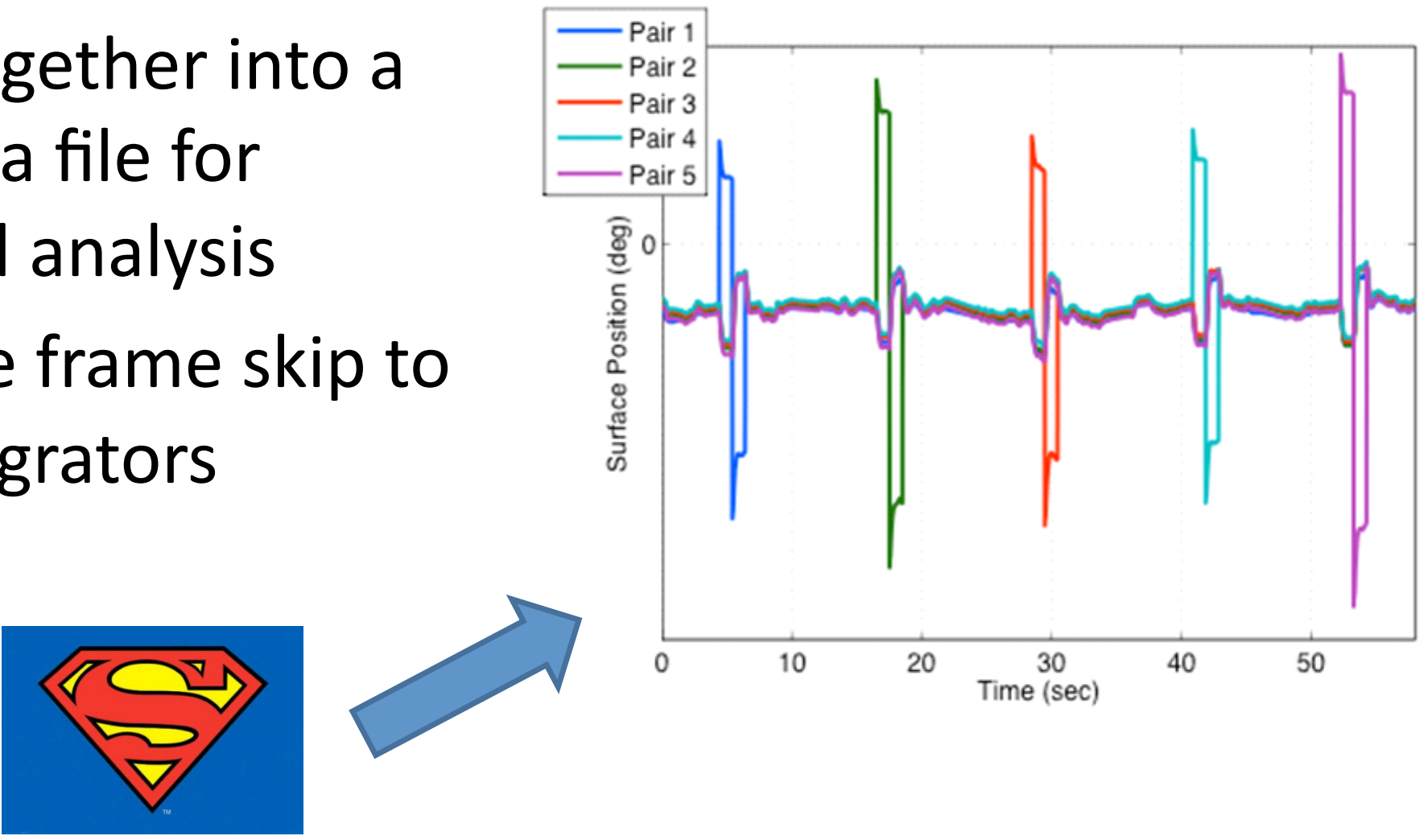

* Not actually a registered trademark, just a cool name for something really simple. 


\section{Data Sources}

- Simulation

- Boeing nonlinear sim v4.3, VMS v4.3.1, aero model 20091223, implemented in Simulink

- PID/OBES and control allocator modified by DFRC for maneuver definition and forcing open-loop

- Flight

- Flights 65, 66, 67

- Flight data filtered though $3^{\text {rd }}$ Order Butterworth filter, $5 \mathrm{~Hz}$ corner frequency

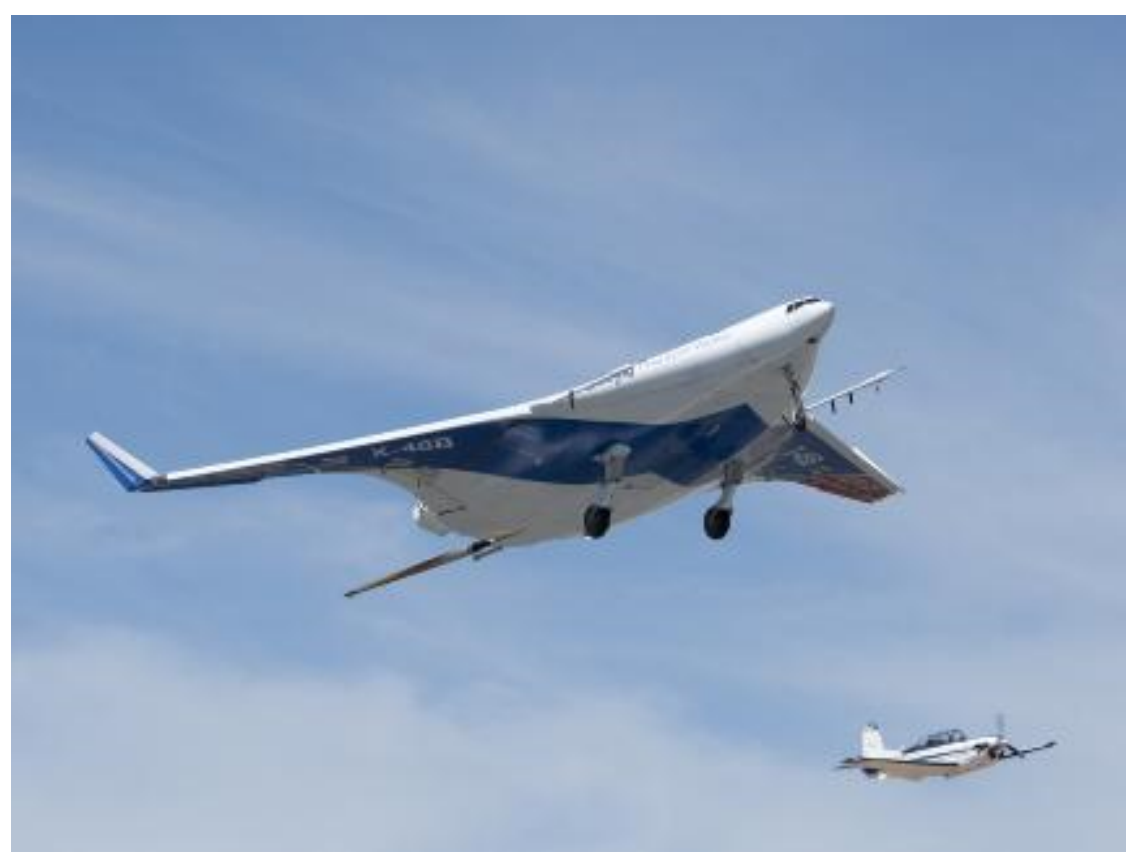

NASA / Tony Landis (ED10-0056-32) 


\section{Linear Regression for PID}

- Model form is assumed to be linear (though regressors may be nonlinear)

- Model composition is

Outputs $=$ Regressors $*$ Parameters

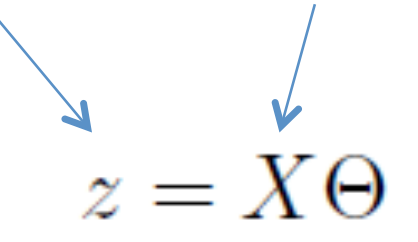

$\hat{\Theta}=\left(X^{T} X\right)^{-1} X^{T} z$ determined from stepwise regression

$$
C_{l}=C_{l o}+C_{l \beta} \cdot \beta+C_{l p} \cdot \frac{p b}{2 V}+C_{l r} \cdot \frac{r b}{2 V}+\left[C_{l, s u r f a c e s}\right]
$$

- Try stuff out and pick one that has low fit error and high $r^{2}$

- Estimate uncertainty from

$$
r^{2}=\frac{\hat{\Theta}^{T} X^{T} z-N \bar{z}^{2}}{z^{T} z-N \bar{z}^{2}}
$$
Cramér-Rao bounds

$$
s=\sqrt{\frac{\sum\left(z_{i}-\hat{y}_{i}\right)^{2}}{N-p}}
$$




\section{Model Structure}

$C_{l}=C_{l, a e r o}+\left(\sum_{j=2}^{5} C_{l \delta a_{j}} \cdot \delta a_{j}\right)+\left(C_{l \delta a i} \cdot \delta a i\right)+\left(C_{l \delta a o} \cdot \delta a o\right)+\left(C_{l \delta d i} \cdot \delta d i\right)+\left(C_{l \delta d o} \cdot \delta d o\right)+\left(C_{l \delta r} \cdot \delta r\right)$

$C_{n}=C_{n, a e r o}+\left(\sum_{j \models 2}^{5} C_{n \delta a_{j}} \cdot \delta a_{j}\right)+\left(C_{n \delta a i} \cdot \delta a i\right)+\left(C_{n \delta a o} \cdot \delta a o\right)+\left(C_{n \delta d i} \cdot \delta d i\right)+\left(C_{n \delta d o} \cdot \delta d o\right)+\left(C_{n \delta r} \cdot \delta r\right)$

$C_{Y}=C_{y, a e r o}+\left(\sum_{j=2}^{5} C_{Y \delta a_{j}} \cdot \delta a_{j}\right)+\left(C_{Y \delta a i} \cdot \delta a i\right)+\left(C_{Y \delta a o} \cdot \delta a o\right)+\left(C_{Y \delta d i} \cdot \delta d i\right)+\left(C_{Y \delta d o} \cdot \delta d o\right)+\left(C_{Y \delta r} \cdot \delta r\right)$

- Abstracted effector definitions, not treating outer surfaces literally.

- Combined axis regressors (same regressor set for logintudinal and lateraldirectional)

\begin{tabular}{|c|c|c|c|}
\hline Model & Structure & $r^{2}$ & $s$ \\
\hline \hline$C_{Y}$ & Combined axes / abstracted effectors & $\mathbf{9 5 . 0 3 \%}$ & $\mathbf{1 7 . 4 2 \%}$ \\
\hline & Combined axes / individual surfaces & $94.06 \%$ & $17.54 \%$ \\
\hline & Split axes / abstracted effectors & $93.20 \%$ & $20.52 \%$ \\
\hline$C_{l}$ & Combined axes / abstracted effectors & $\mathbf{9 0 . 2 2 \%}$ & $\mathbf{3 1 . 2 1 \%}$ \\
\hline & Combined axes / individual surfaces & $89.59 \%$ & $32.21 \%$ \\
\hline & Split axes / abstracted effectors & $76.90 \%$ & $48.05 \%$ \\
\hline$C_{n}$ & Combined axes / abstracted effectors & $\mathbf{9 3 . 7 9 \%}$ & $\mathbf{2 4 . 7 7 \%}$ \\
\hline & Combined axes / individual surfaces & $93.23 \%$ & $25.87 \%$ \\
\hline & Split axes / abstracted effectors & $90.67 \%$ & $30.17 \%$ \\
\hline
\end{tabular}




\section{Inner Surfaces}

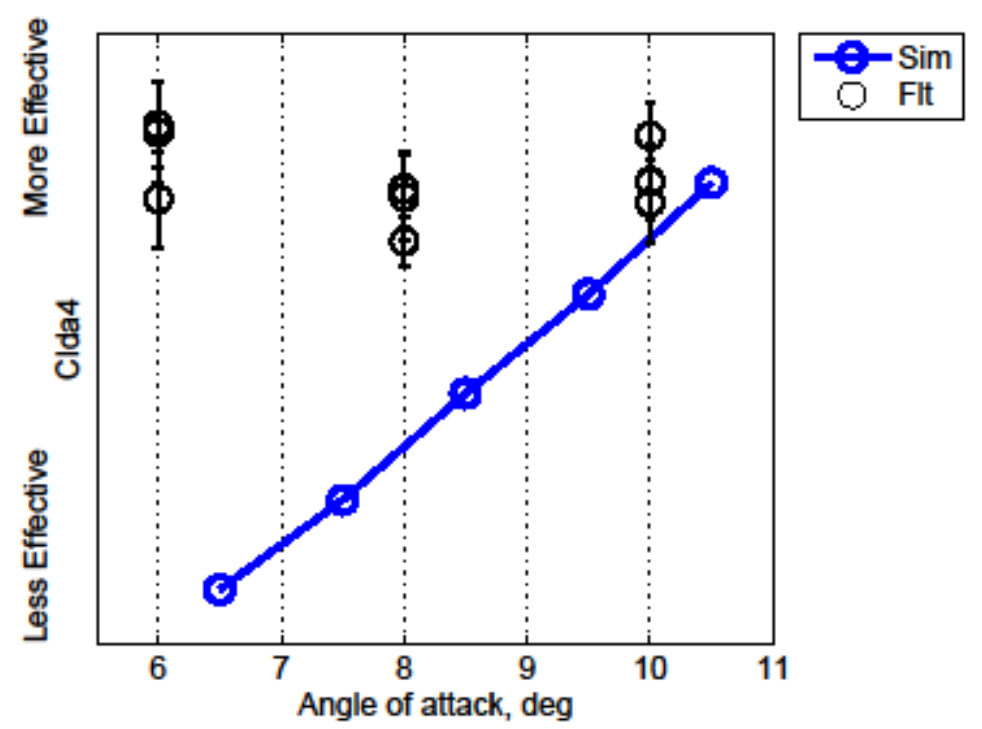

- Model is overall quite consistent

- Simulation estimates shown for anchoring, not comparison

- Bands are CRB with colored noise assumption
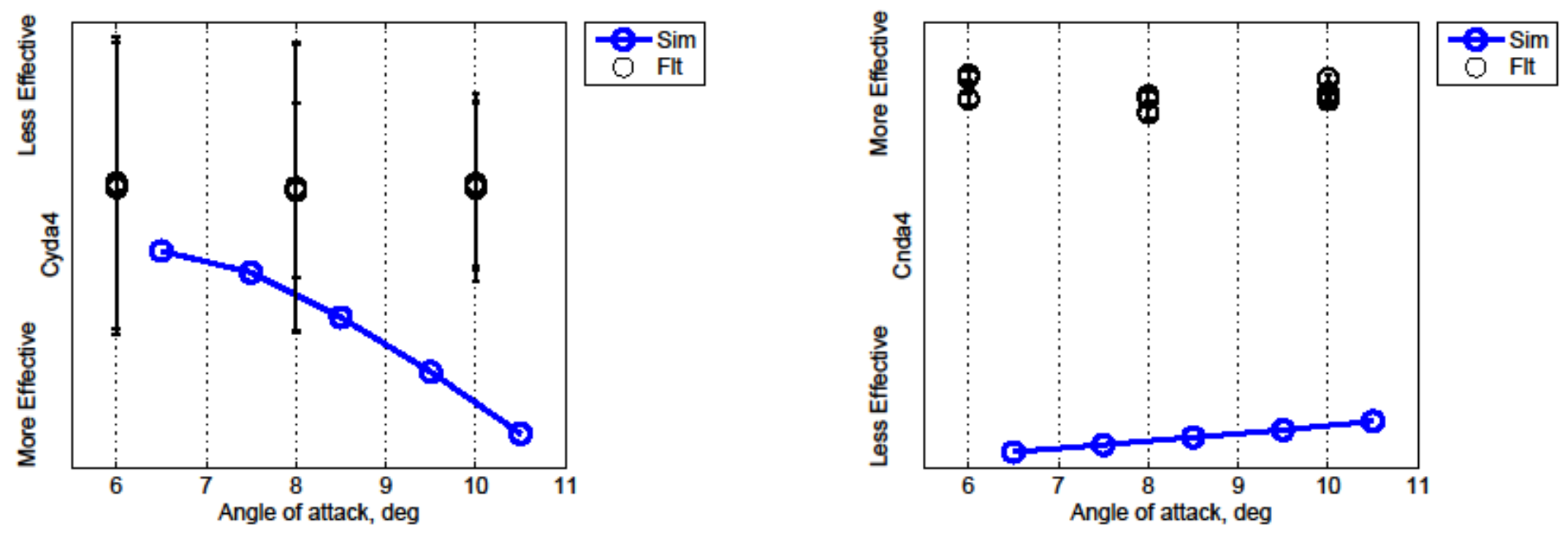


\section{Abstracted Effectors ( $\delta a i)$}

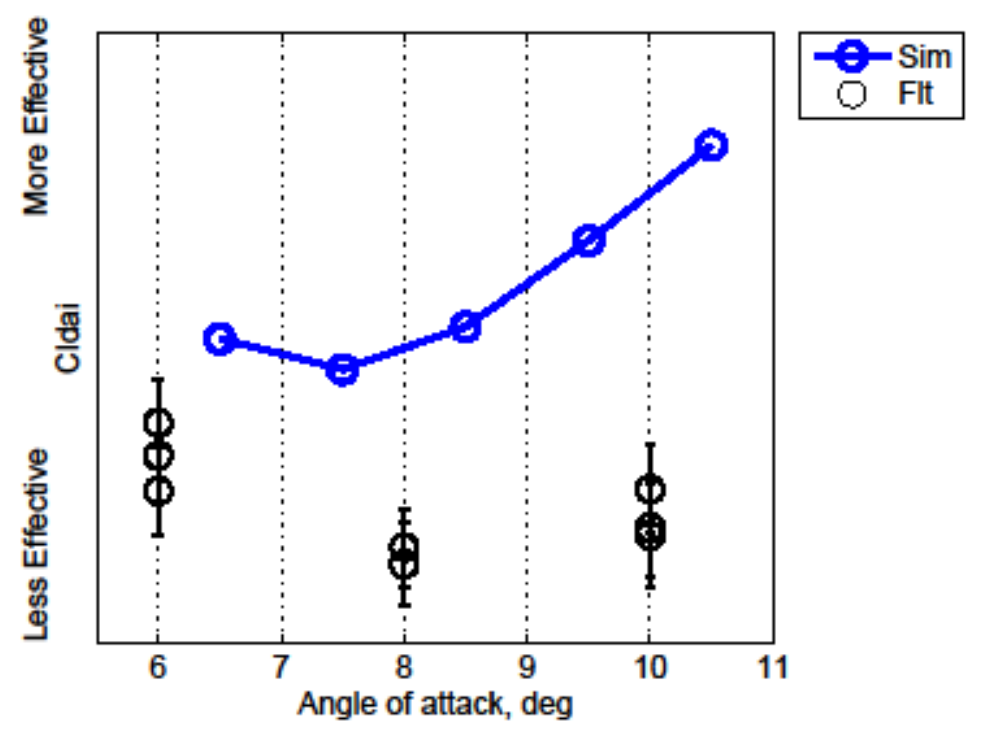

- Aileron-like clamshell movement shows good parameter consistency

- Off-axis derivatives are well-captured
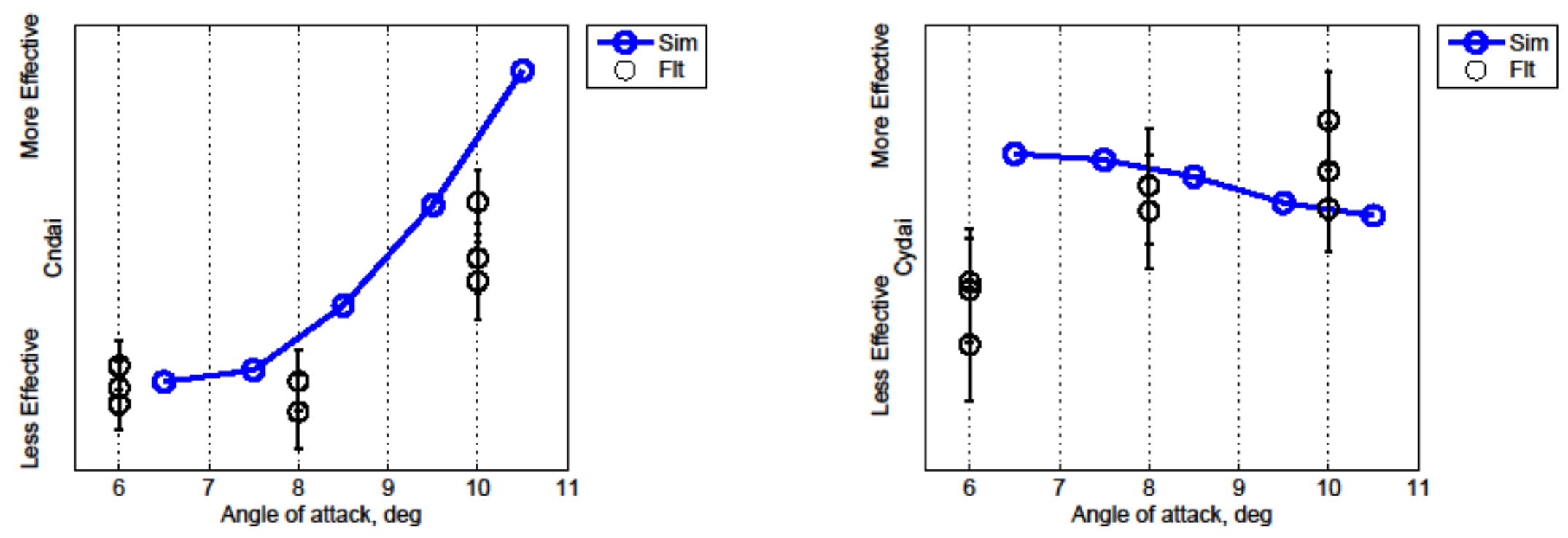


\section{Abstracted Effectors ( $\delta d o$ )}

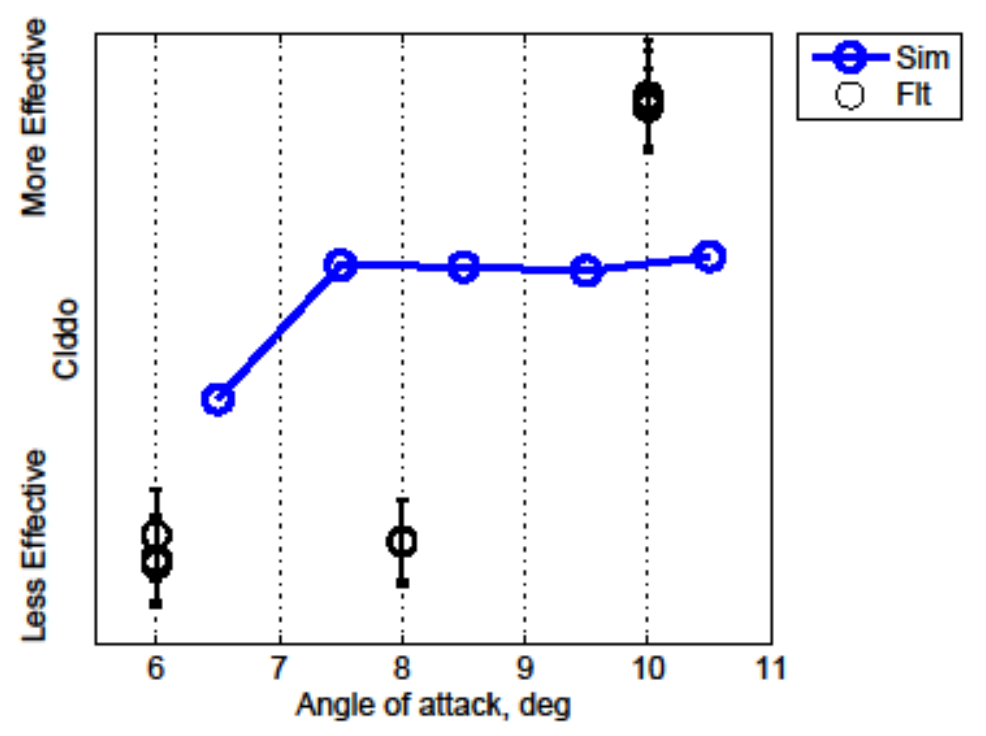

- Drag rudder yaw effector shows excellent parameter consistency

- Off-axis derivatives are well-captured
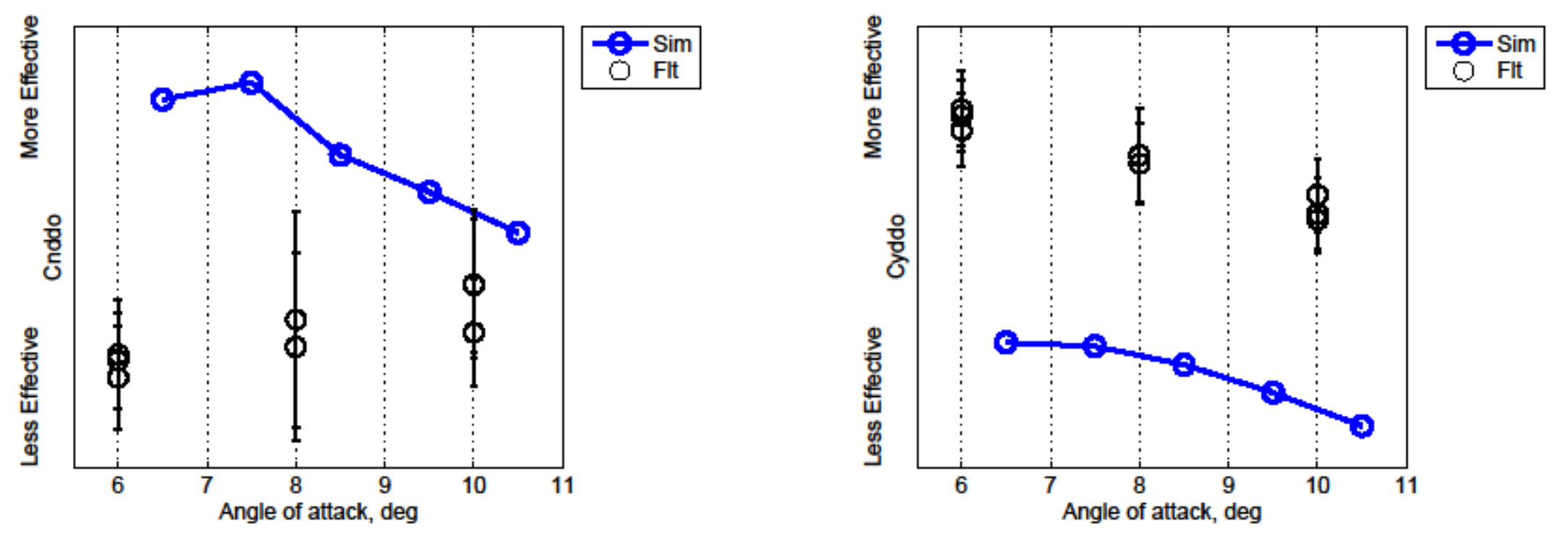


\section{Overall Model Fit Accuracy}

- Can the models reconstruct observed state variable time histories from same applied inputs?

- Test on sim data first

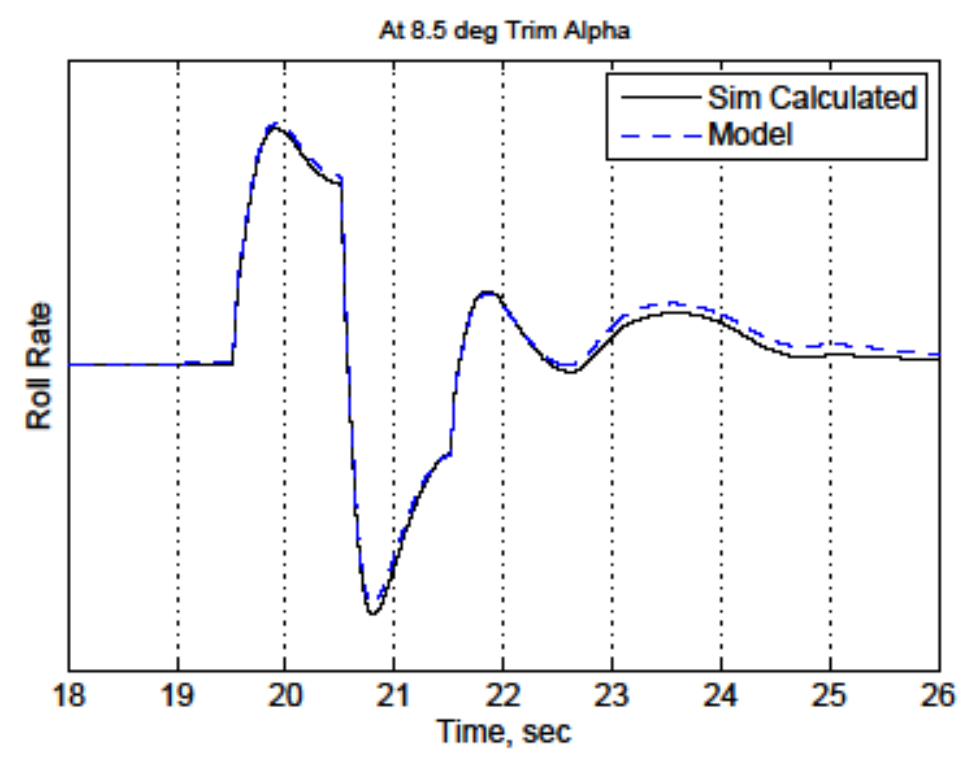

$$
\begin{aligned}
& \dot{\beta}=\frac{\bar{q}_{E} S}{m V_{E}} C_{Y}+p \sin \alpha_{E}-r \cos \alpha_{E}+\frac{g}{V_{E}} \sin \phi_{E} \cos \theta_{E} \\
& \dot{p}-\frac{I_{x z}}{I_{x x}} \dot{r}=\frac{\bar{q}_{E} S b}{I_{x x}} C_{l}-\frac{\left(I_{z z}-I_{y y}\right)}{I_{x x}} q_{E} r+\frac{I_{x z}}{I_{z z}} q_{E} p \\
& \dot{r}-\frac{I_{x z}}{I_{z z}} \dot{p}=\frac{\bar{q}_{E} S b}{I_{z z}} C_{n}-\frac{\left(I_{y y}-I_{x x}\right)}{I_{z z}} q_{E} p+\frac{I_{x z}}{I_{z z}} q_{E} r
\end{aligned}
$$




\section{Overall Model Fit Accuracy}

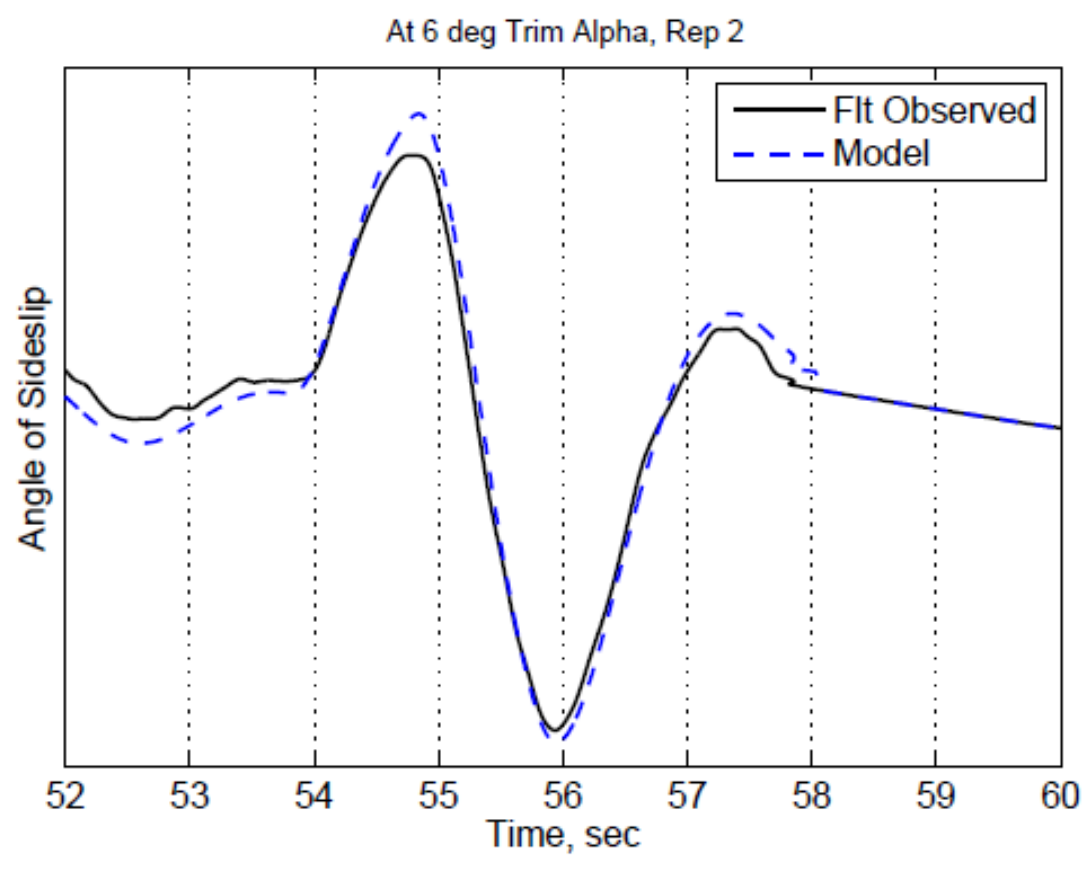

(a) Angle of sideslip, $\alpha_{\text {trim }}=6 \mathrm{deg}$.

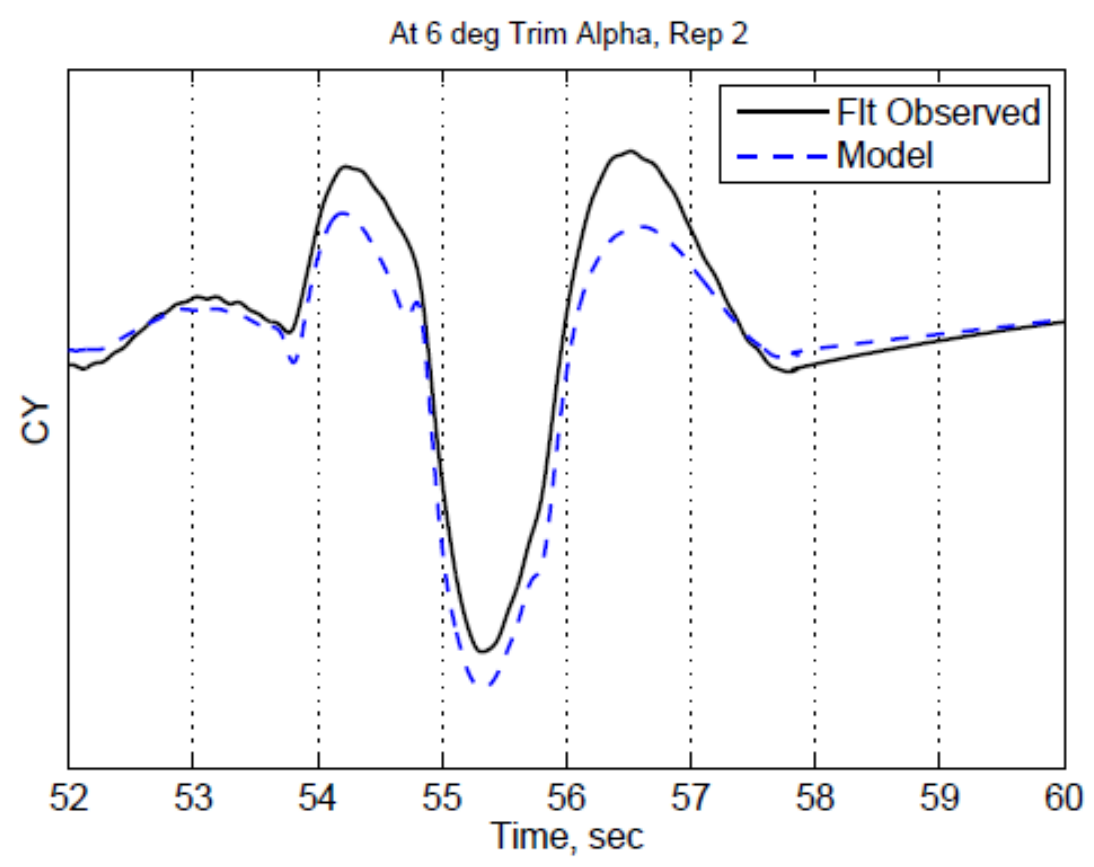

(b) Coefficient of side force, $\alpha_{\text {trim }}=6 \mathrm{deg}$.

Flight data is reconstructed well by the model. 


\section{Overall Model Fit Accuracy}

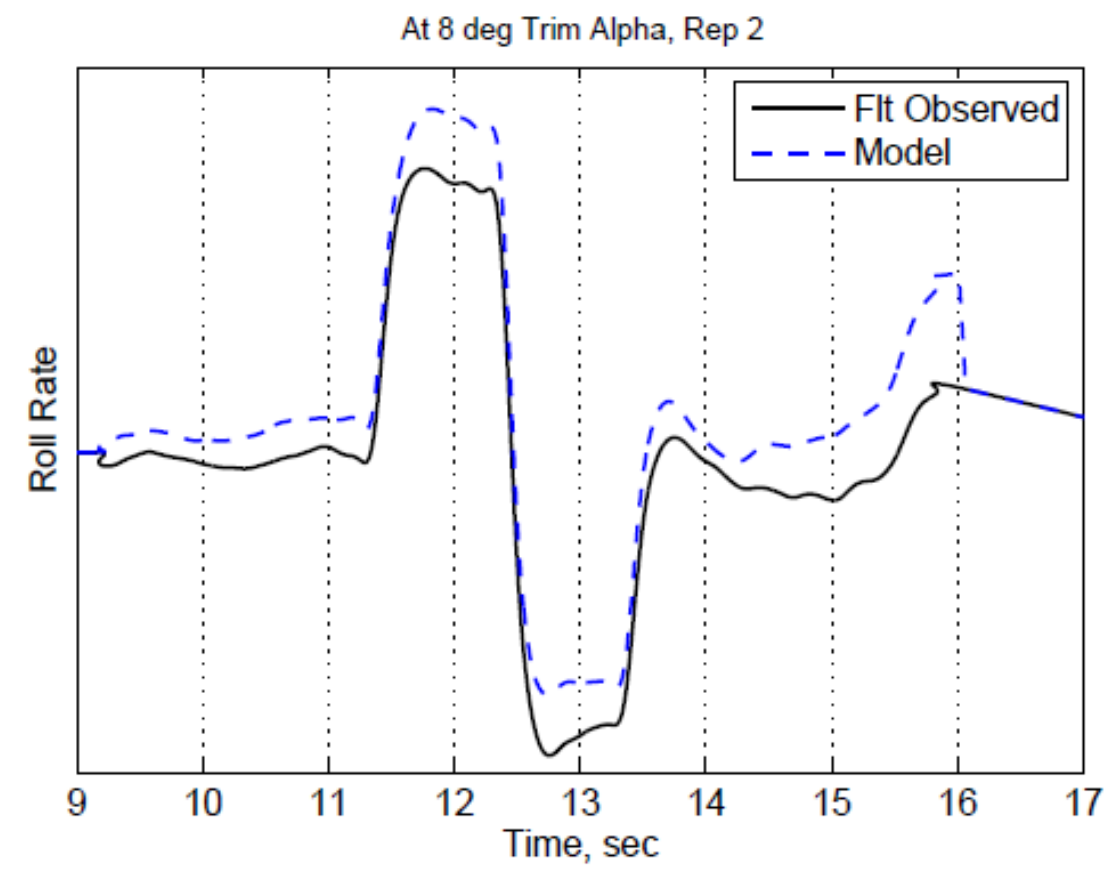

(a) Roll rate, $\alpha_{\text {trim }}=8 \mathrm{deg}$.

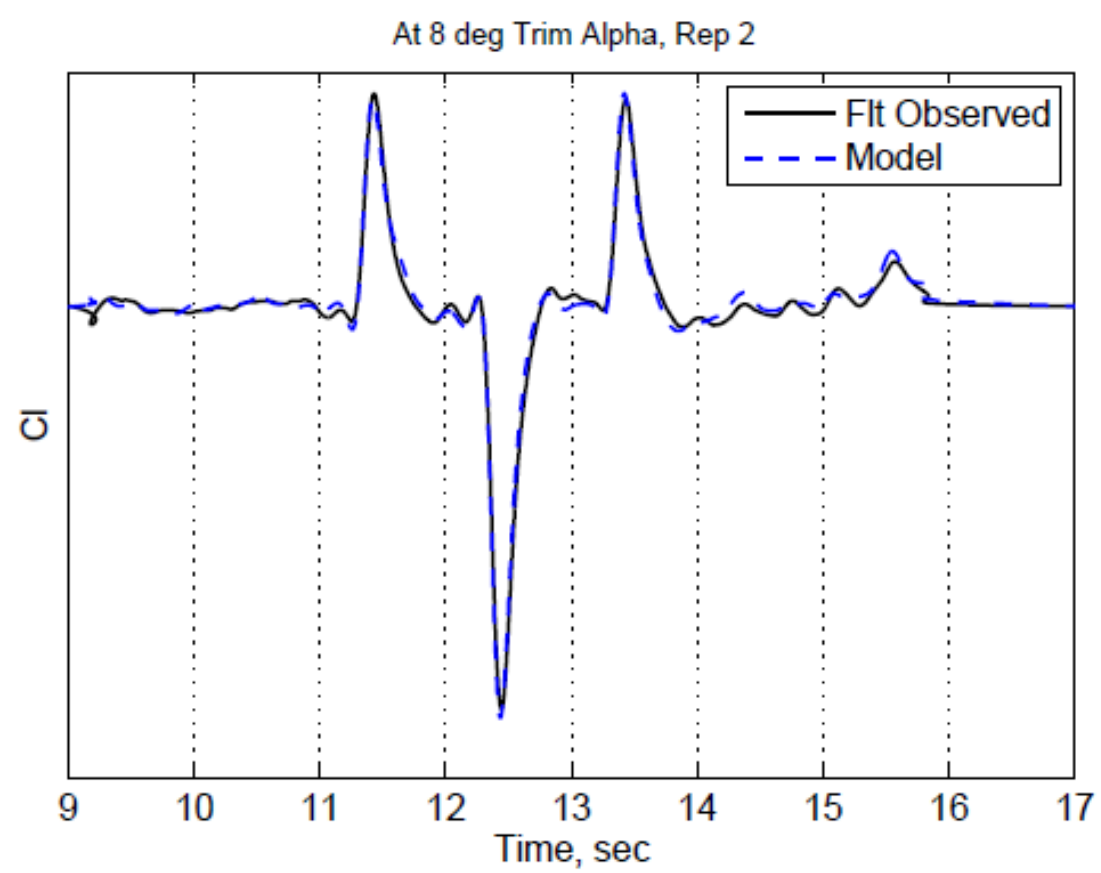

(b) Coefficient of rolling moment, $\alpha_{\text {trim }}=8 \mathrm{deg}$.

Flight data is reconstructed well by the model. 


\section{Overall Model Fit Accuracy}

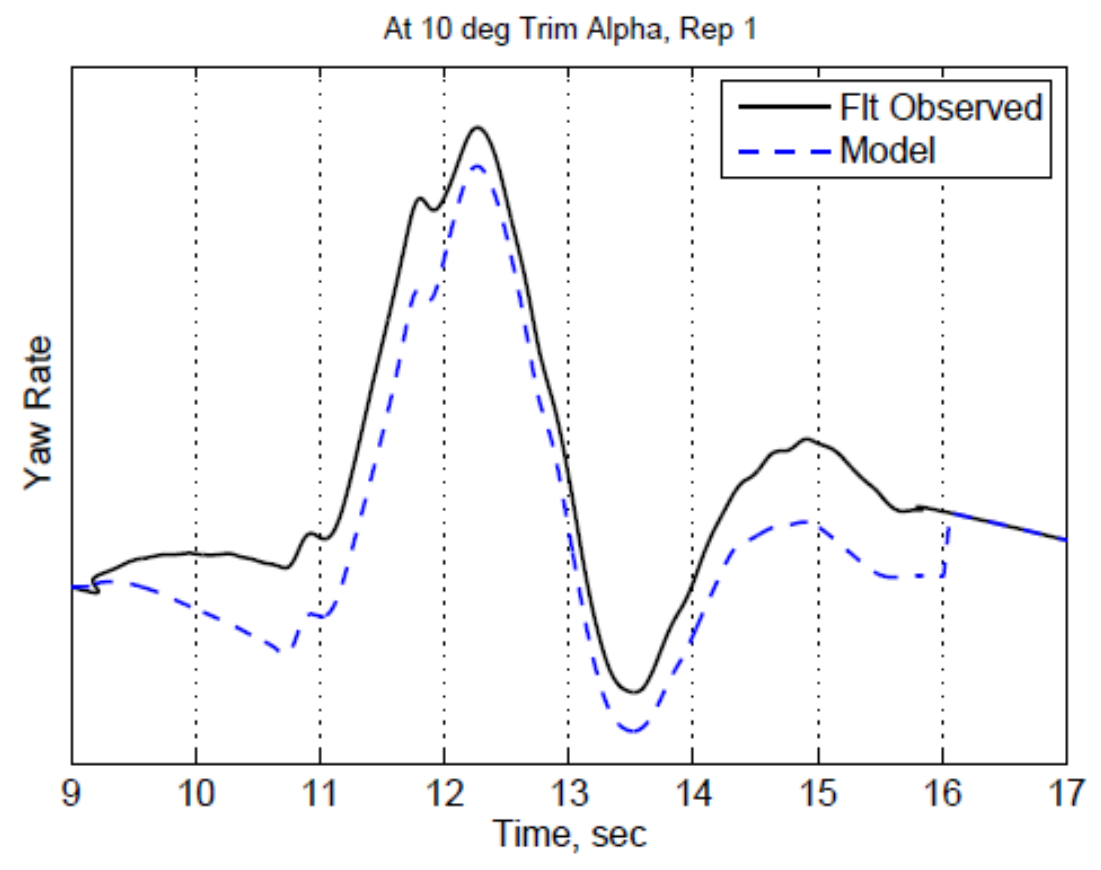

(a) Yaw rate, $\alpha_{\text {trim }}=10 \mathrm{deg}$.

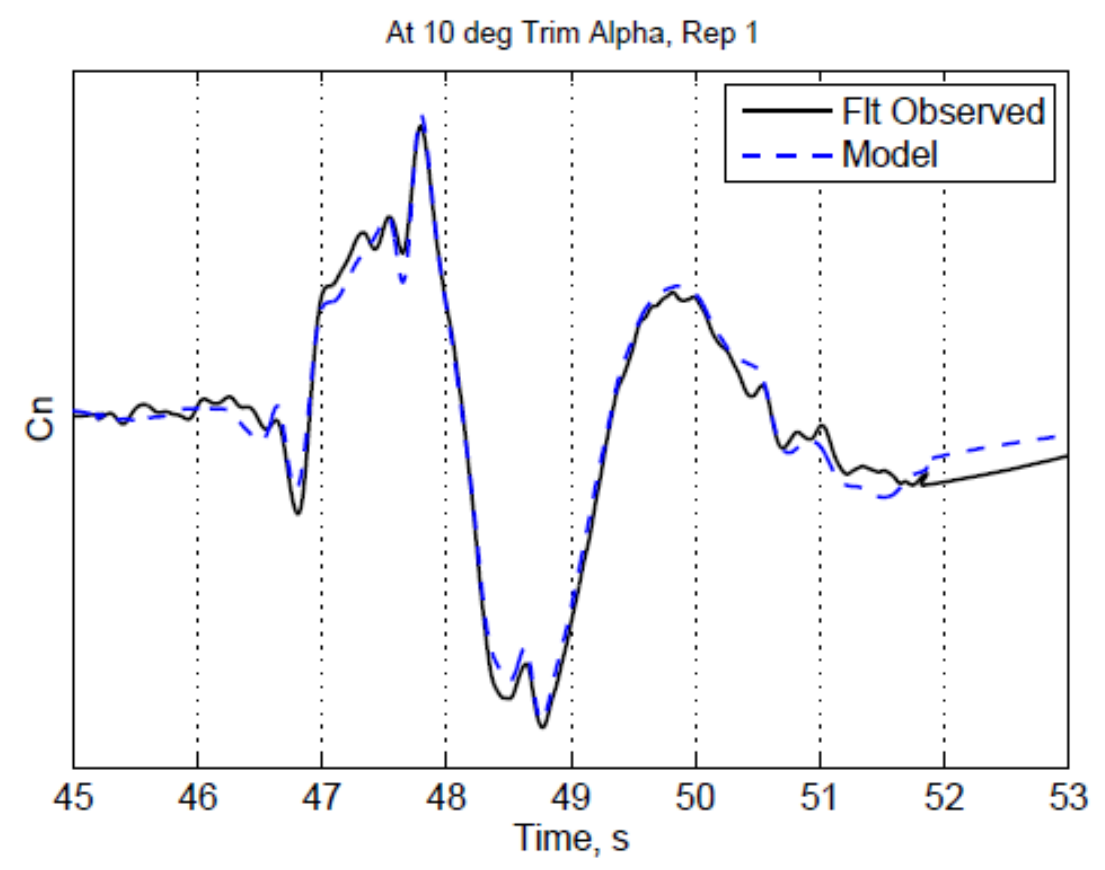

(b) Coefficient of yawing moment, $\alpha_{\text {trim }}=10 \mathrm{deg}$.

Flight data is reconstructed well by the model. 


\section{Conclusions}

- Stepwise regression was found to be a useful technique for lateral-directional model design for HWB aircraft.

- Linear regression parameter estimation methods using abstracted effectors may be expected to perform well (delivering consistent, accurate, and high-confidence estimates) for HWB aircraft.

- Accounting for cross-axis dynamics improves model fidelity despite the increased complexity of the regression equation for HWB.

- Defining mathematical abstractions for complex surfaces that more accurately capture the effective total input seen by the system can result in higher model fidelity than treating the physical surface motion literally. 


\section{Future Work}

- Combined multi-axis parameter estimates using, asymmetric, single-surface PID maneuvers

- De-correlation using orthogonal Walsh function inputs on all surfaces simultaneously

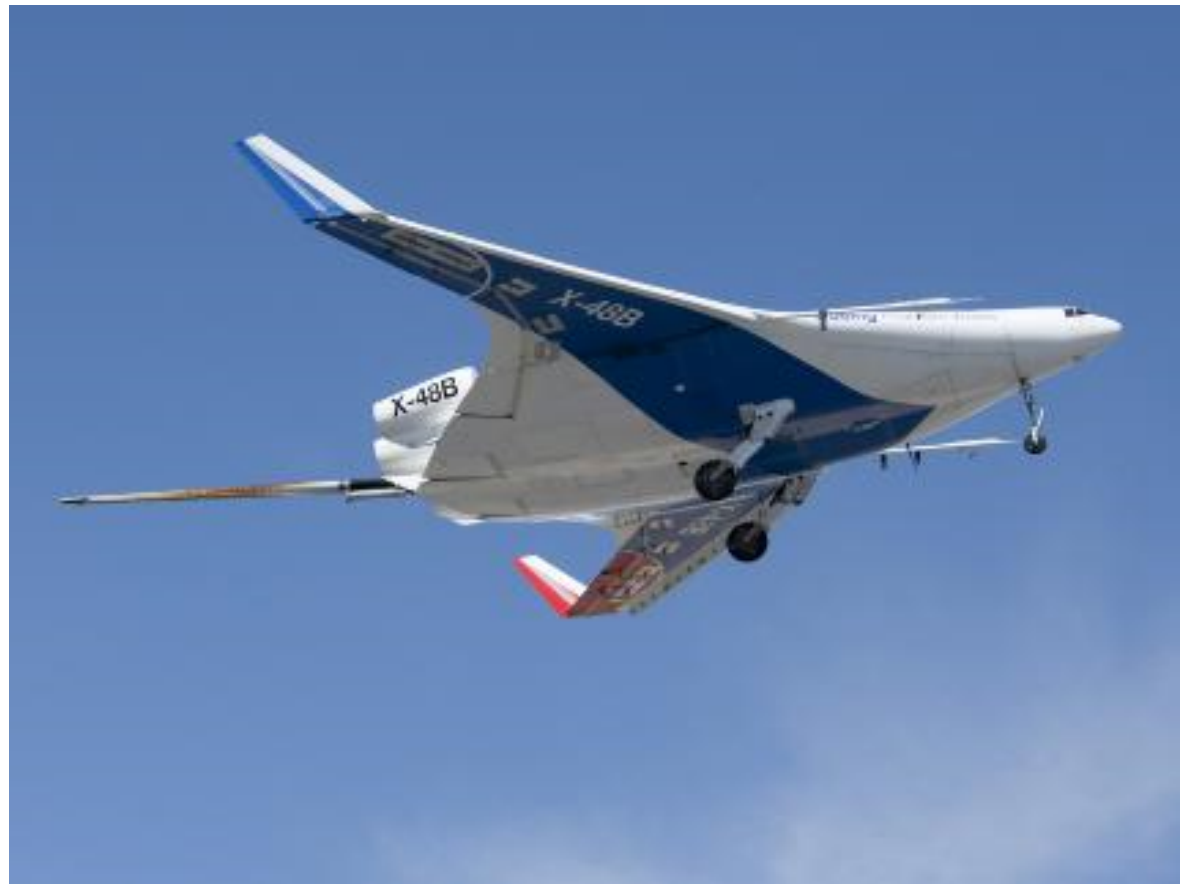

NASA / Tony Landis (ED10-0056-35) 


\section{Questions?}
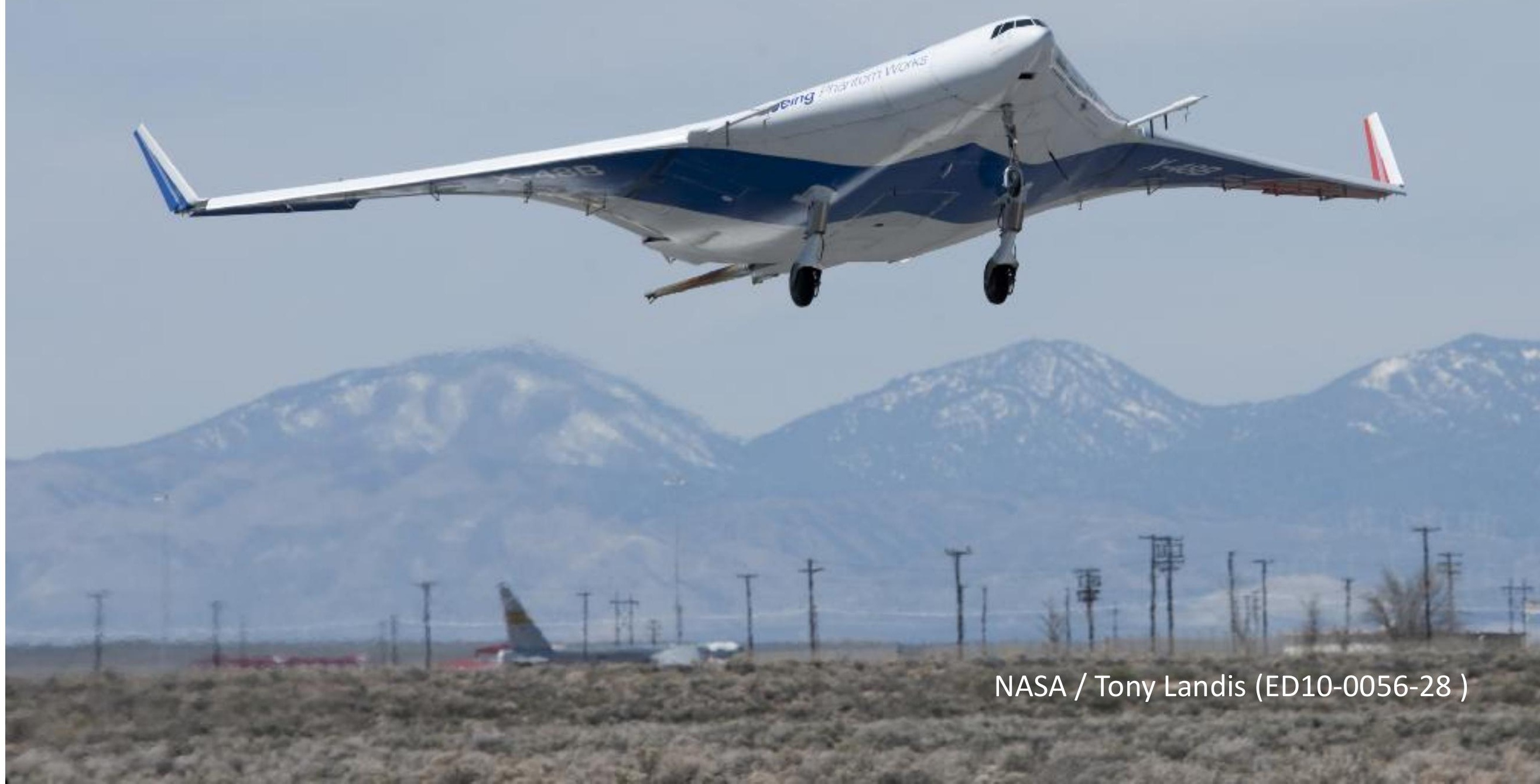